\title{
¿DIFERENCIAS CONDUCTUALES ENTRE NEANDERTALES Y HUMANOS MODERNOS?: EL CASO DEL PALEOLÍTICO MEDIO EN EL PRÓXIMO ORIENTE
}

\author{
BEHAVIORAL DIFFERENCES BETWEEN NEANDERTALS AND MODERN HUMANS?: \\ THE CASE OF THE MIDDLE PALEOLITHIC IN THE NEAR EAST
}

IGNACIO DE LA TORRE SÁINZ $\left(^{*}\right)$
MANUEL DOMÍNGUEZ-RODRIGO $\left.{ }^{*}\right)$

A la memoria de Gonzalo Muñoz.

\section{RESUMEN}

La región del Próximo Oriente es única para el estudio de las diferencias conductuales entre neandertales y humanos modernos, pues aparecen juntos en un restringido espacio temporal y en un rango cronológico definido, el Pleistoceno superior inicial. En los últimos años, se han propuesto varias hipótesis sobre las posibles distinciones en el comportamiento de unos y otros homínidos. En el presente estudio se analizan las conclusiones obtenidas por los investigadores que trabajan en la zona, y se replantean las teorías que abogan por una diferenciación conductual entre los neandertales y los humanos anatómicamente modernos del Próximo Oriente.

\begin{abstract}
The Near East region is unique for the study of the behavior of Neandertals and early modern humans, since both types of hominids appear together in the same space within a limited time framework (Early Upper Pleistocene). In recent years, several hypotheses have been proposed to explain differential behaviors in these hominids. In this work, the conclusions obtained by different researchers in this regard are analyzed. The theories arguing for different behaviors between neandertals and modern humans are revised critically.
\end{abstract}

Palabras clave: Próximo Oriente. Pleistoceno superior inicial. Paleolítico Medio. Neandertales. Primeros humanos modernos.

(*) Dpto. de Prehistoria, Facultad de Geografía e Historia, Universidad Complutense. Ciudad Universitaria, s/n $28040 \mathrm{Ma}-$ drid. Correo electrónico: itorresainz@ terra.es

El artículo fue remitido en su versión final el 7-XI-2000.
Key words: Near East. Early Upper Pleistocene. Middle Paleolithic. Neandertals. First anatomically modern humans.

\section{INTRODUCCIÓN}

Hasta la fecha, sólo dos zonas del registro euroasiático aportan evidencias de una posible convivencia e interrelación entre los humanos modernos y los neandertales. Una es la región de Europa occidental, donde se documenta una coexistencia de unos neandertales tardíos, asociados a una industria leptolítica y a una cultura del Paleolítico superior, junto a humanos anatómicamente modernos que, con sus industrias auriñacienses, terminarían por sustituir a los grupos autóctonos europeos. Sin embargo, tal coexistencia se redujo a unos pocos milenios; hacia el 40.000 BP tenemos en el área las primeras evidencias del Auriñaciense, y tan sólo 10-12.000 años después los últimos neandertales se extinguieron en el sur de la Península Ibérica (véanse las síntesis recientes de d'Erricoet alii, 1998; Zilhao y d'Errico, 1999, 2000; Mellars et alii, 1999; Kozlowski y Otte, 2000; Bocquet-Appel y Demars, 2000).

La otra región donde tenemos restos de ambos tipos de homínidos, muchas veces en yacimientos separados por pocos metros, es el Próximo Oriente. En esta zona, el panorama cultural es muy distinto al del registro francocantábrico. En primer lugar, por razones cronológicas: mientras que la posible coexistencia entre modernos y neandertales en Europa tuvo lugar hacia el 40.000 BP, en el Próximo Oriente los humanos modernos ocupaban 


\begin{tabular}{|c|c|c|c|c|c|c|}
\hline Yacimiento/Nivel & Facies & $\mathrm{Th} / \mathrm{U}$ & TL & ESR* & $\mathrm{ESR}^{* *}$ & C-14 \\
\hline Umm el Tlel 4 & $\begin{array}{l}\text { Inter-Yabrudiense- } \\
\text { Musteriense }\end{array}$ & $76 \pm 16$ & & & & \\
\hline $\begin{array}{c}\text { Tabun } \\
\text { Unidad I (=C) } \\
\text { Unidad II (=D) } \\
\text { Unidad V (=D) } \\
\text { Unidad IX (=D) } \\
\text { B } \\
\text { C } \\
\text { D }\end{array}$ & $\begin{array}{l}\text { Musteriense tipo C } \\
\text { Musteriense tipo D } \\
\text { Musteriense tipo D } \\
\text { Musteriense tipo D } \\
\text { Musteriense tipo B } \\
\text { Musteriense tipo C } \\
\text { Musteriense tipo D }\end{array}$ & & $\begin{array}{l}171 \pm 17 \\
212 \pm 22 \\
244 \pm 28 \\
265 \pm 27\end{array}$ & $\begin{array}{c}86 \pm 11 \\
102 \pm 17 \\
122 \pm 20\end{array}$ & $\begin{array}{l}103 \pm 18 \\
119 \pm 11 \\
166 \pm 20\end{array}$ & $\begin{array}{c}39.7 \pm 0.8 \\
40.9 \pm 10\end{array}$ \\
\hline $\begin{array}{l}\text { Hayonim } \\
\text { Superior E } \\
\text { Superior E } \\
\text { Inferior E } \\
\end{array}$ & $\begin{array}{l}\text { Musteriense tipo C } \\
\text { Musteriense tipo C } \\
\text { Musteriense tipo D }\end{array}$ & $163 \pm 3$ & & $\begin{array}{l}164 \pm 15 \\
241 \pm 11 \\
\end{array}$ & $\begin{array}{l}171 \pm 17 \\
257 \pm 6 \\
\end{array}$ & \\
\hline $\begin{array}{c}\text { Qafzeh } \\
\text { XVII-XXII Media } \\
\text { XV-XXI }\end{array}$ & $\begin{array}{l}\text { Musteriense tipo } \mathrm{C} \\
\text { Musteriense tipo } \mathrm{C}\end{array}$ & & Media $92 \pm 5$ & Media $96 \pm 13$ & $115 \pm 15$ & \\
\hline $\begin{array}{c}\text { Kebara } \\
\text { Unidad VI } \\
\text { Unidad VII } \\
\text { Unidad VIII } \\
\text { Unidad IX } \\
\text { Unidad X } \\
\text { Unidad XI } \\
\text { Unidad XII } \\
\text { Nivel B } \\
\text { Nivel B }\end{array}$ & Musteriense tipo B & & $\begin{array}{l}48.3 \pm 3.5 \\
51.9 \pm 3.5 \\
57.3 \pm 4.0 \\
58.4 \pm 4.0 \\
61.6 \pm 3.6 \\
60.0 \pm 3.5 \\
59.9 \pm 3.5\end{array}$ & $60 \pm 6$ & $64 \pm 4$ & $\begin{array}{c}41 \pm 10 \\
35.3 \pm 0.5\end{array}$ \\
\hline Quneitra & Musteriense final & & & $39.2 \pm 4.2$ & $53.9 \pm 5 . .9$ & \\
\hline Skhul & Musteriense tipo C & & Media $119 \pm 18$ & $81 \pm 15$ & $101 \pm 12.6$ & \\
\hline $\begin{array}{c}\text { Amud } \\
\text { B } 1 / 6 \\
\text { B2/8 } \\
\text { B4 } \\
\end{array}$ & Musteriense tipo B & & $\begin{array}{l}57.6 \pm 3.7 \\
56.5 \pm 3.5 \\
68.5 \pm 3.4\end{array}$ & $\begin{array}{c}43 \pm 5 \\
59 \pm 8 \\
68\end{array}$ & $\begin{array}{c}48 \pm 6 \\
70 \pm 8 \\
113 \pm 8 \\
\end{array}$ & \\
\hline Far'ah II & Musteriense tipo B & $72.5 \pm 1.5$ & & $49.1 \pm 4.1$ & $62.2 \pm 7.0$ & \\
\hline Ksar Akil & Musteriense final & & & & & $42.75 \pm 1.5$ \\
\hline $\begin{array}{ll} & \text { Shanidar } \\
D & (\mathrm{GrN}-2527) \\
D & (\mathrm{GrN}-1495)\end{array}$ & $\begin{array}{l}\text { Musteriense tipo } \\
\text { Zagros }\end{array}$ & & & & & $\begin{array}{c}46.8 \pm 1.5 \\
50.6 \pm 30\end{array}$ \\
\hline \begin{tabular}{lc}
\multicolumn{2}{c}{ Boker Tachtit } \\
Nivel 1 & (GX-3642) \\
Nivel 1 & (SMU-580) \\
Nivel l & (SMU-259) \\
Nivel 1 & (SMU-184) \\
Nivel 4 & (SMU-579) \\
\end{tabular} & $\begin{array}{c}\text { Musteriense final } \\
\mathrm{e} \\
\text { Industrias } \\
\text { transicionales }\end{array}$ & & & & & $\begin{array}{c}>35.000 \\
47.28 \pm 9.05 \\
44.93 \pm 2.42 \\
>45.490 \\
35.055 \pm 4.1 \\
\end{array}$ \\
\hline
\end{tabular}

Tab. 1. Cronología de los principales yacimientos musterienses del Próximo Oriente, a partir de la síntesis de dataciones en de la Torre y Domínguez-Rodrigo (2000). *Absorción rápida del Uranio. **Absorción paulatina del Uranio.

el área ya en el último interglacial, y se piensa que los neandertales llegaron después a la región, coincidiendo con los comienzos delWürm europeo. De este modo, y si asumiéramos que hubo una coexistencia entre ambos grupos a lo largo del Pleistoceno superior inicial, ésta duró nada menos que
50.000 años. Además, los dos grupos humanos compartieron una tecnología prácticamente idéntica, ocuparon los mismos asentamientos, y cazaron los mismos animales. No obstante, la interrelación entre humanos anatómicamente modernos y neandertales es difícil de probar. En todos los yacimien-

T. P., 58, n. ${ }^{\circ} 1,2001$ 
tos con industrias del llamado Musteriense tipo Tabun B en los que se han encontrado restos humanos, éstos han sido identificados como neandertales, y datan de comienzos del Würm. Por su parte, los yacimientos asociados a humanos modernos, los del Musteriense tipo Tabun C, se sitúan entre el 170.000 y el 100.000 BP (Tab. 1), por lo que podrían haber sido sustituidos en el área por los neandertales tras el último interglacial. Hubiera o no interrelación entre ambos grupos de homínidos, lo cierto es que la similitud de sus industrias, la cercanía de los asentamientos y la cronología parecida que manejamos, permiten establecer comparaciones entre el comportamiento de unos y de otros.

El Paleolítico del Próximo Oriente ha sido objeto de la dedicación de numerosos investigadores desde comienzos de este siglo. No obstante, pocos autores han tratado de comparar los restos asociados a ambos tipos de homínidos, y establecer así hipótesis acerca de la ocupación del territorio por parte de estos grupos, las posibles diferencias económicas, de asentamiento, etc. Sólo en los últimos años, el cambio de perspectiva de algunos investigadores ha permitido la elaboración de interesantes trabajos sobre el tema. De este modo, se ha intentado establecer modelos de comportamiento dicotómicos entre ambos grupos de homínidos en función de la fauna representada en los yacimientos (Lieberman, 1993), de la industria (Shea, 1995, 1998), e incluso a partir de los propios restos humanos (Trinkaus, 1989, 1992). Sin embargo, y a pesar de estos innovadores estudios, la interpretación de los yacimientos levantinos y de la interrelación entre humanos modernos y neandertales sigue estando dominada por asunciones apriorísticas e hipótesis erróneas.

Tras haber presentado en un trabajo anterior la secuencia cronoestratigráfica y cultural del Pleistoceno superior inicial de la región (de la Torre y Domínguez-Rodrigo, 2000), en las páginas siguientes intentaremos demostrar que los datos disponibles no permiten establecer modelos diferentes de comportamiento para humanos modernos y neandertales en el Próximo Oriente. Para ello, analizaremos la industria y la fauna de los distintos yacimientos, en busca de posibles diferencias en las estrategias económicas de ambos taxones, estudiando luego el abundante registro antropológico, que nos aportará información sobre la anatomía y comportamiento simbólico de los habitantes de la región durante el Pleistoceno medio final y los comienzos del Würm.

\section{LA DIVISIÓN DEL MUSTERIENSE Y SU ADSCRIPCIÓN A LOS TIPOS HUMANOS}

El Paleolítico medio del Próximo Oriente se estructura a partir de las facies industriales estudiadas en el yacimiento de Tabun. Garrod (Garrod y Bate, 1937) diferenció una facies antigua, que denominó Levalloiso-Musteriense inferior en función de los materiales del nivel D de esta cueva. Por encima de dicha unidad, identificó una industria ligeramente distinta, más evolucionada, que Garrod calificó de Levalloiso-Musteriense superior (niveles C y B de Tabun). Décadas más tarde, Copeland (1975) sistematizó todo el Paleolítico medio de la región proponiendo la identificación de tres facies sucesivas. Así, esta autora diferenció el llamado Musteriense tipo Tabun D, el tipo Tabun C y el tipo Tabun B. Tanto las hipótesis de Copeland (1975, 1981, 1983) como las de Jelinek $(1981,1982)$ y otros autores estaban basadas en una concepción evolutiva unilineal de las industrias. De este modo, se pensaba que en su mayor parte el Musteriense se asociaba con los neandertales (excepción hecha de Qafzeh y Skhul), quienes evolutivamente darían lugar a los Homo sapiens sapiens. No obstante, en esos mismos años algunos investigadores comenzaron a proponer que los humanos modernos ocupaban el área antes que los neandertales (Bar-Yosef y Vandermeersch, 1981). Según esta hipótesis, la sucesión de las facies del Paleolítico medio de la región no podía ser unilineal, sino por sustitución de unas especies por otras. De hecho, hoy se identifica cada tradición musteriense con un tipo diferente de homínido y una cronología distinta. Según las convenciones actuales, podemos diferenciar en la zona costera del Próximo Oriente las siguientes facies (véase con más detalle en de la Torre y Domínguez-Rodrigo, 2000):

- El Musteriense tipo Tabun D: Se observa una mínima preparación de las plataformas de lascado, de las que se extraen como productos láminas y puntas alargadas. En general, los conjuntos contienen porcentajes mayores de piezas retocadas que en las facies posteriores. Las dataciones de Tabun (Mercier et alii, 1995) y Hayonim (Schwarcz y Rink, 1998), sugieren que esta industria se desarrolló en el Próximo Oriente entre el 250.000 y el 170.000 BP.

- El Musteriense tipo Tabun C: los productos son ovalares y rectangulares, no tan laminares como en el tipo Tabun D, y se obtienen a partir de núcleos Levallois con preparación centrípeta y/o bidireccio- 
nal, apareciendo además las puntas aparecen ahora sólo de manera ocasional. Esta industria cubre un rango cronológico amplio, tal y como sugieren las dataciones de Tabun, cuyo nivel C oscila entre los 170.000 (Mercier et alii, 1995) y los 100.000 años (Grün et alii, 1991), de Hayonim nivel E superior (Schwarcz y Rink, 1998), situado hacia el 170.000 BP, y las de Qafzeh (Valladas et alii, 1988) y Skhul (Mercier et alii, 1993), entre el 120-90.000 BP.

- El Musteriense tipo Tabun B: Los productos comunes son las puntas Levallois de base ancha, aunque también se obtienen láminas y lascas. Yacimientos comoAmud (Valladas et alii, 1999) o Kebara (Valladas et alii,1987) no superan los 7060.000 años, mientras que el nivel B de Tabun oscila entre el 100.000 y el 80.000 BP (Grün et alii, 1991).

Al haberse encontrado restos de neandertales en Kebara, Tabun B, Amud y Dederiyeh asociados al Musteriense tipoTabun B, y de humanos modernos en Qafzeh y Skhul (con industria tipo Tabun C), se concluye que todos los yacimientos con industrias de tipo $B$ fueron ocupados por los neandertales, y que los de la fase $\mathrm{C}$ fueron habitados por los humanos anatómicamente modernos (Bar-Yosef, 1992, 1994, 1998; Lieberman, 1993; Lieberman y Shea, 1994; Shea, 1998; Nishiaki, 1998; Goren-Inbar y BelferCohen, 1998, etc). Esta convención académica es fundamental para el objetivo de este trabajo: la comparación entre la conducta de ambos taxones. No obstante, el modelo tiene algunos problemas, como por ejemplo el debate acerca de la posición estratigráfica deTabun I (ver Bar-Yosef y Callander, 1999; Quam y Smith, 1998; Trinkaus, 1993; Trinkaus y Ruff, 1999; Rak, 1998; Schwarczet alii, 1998; Grün y Stringer, 2000; Millard y Pike, 1999;Alperson et alii, 2000). En este yacimiento, se recuperaron en el nivel C una mandíbula (Tabun II o Tabun C2), atribuida hoy aHomo sapiens sapiens (Vandermeersch, 1981; Quam y Smith, 1998; Rak, 1998, etc, en contra Trinkaus 1993) y el cuerpo casi completo deTabun I (tambiénTabun C1), una mujer neandertal.Tabun I, adscrito al nivel C, se encontraba muy cerca del estrato B, por lo que la propia Garrod (Garrod y Bate, 1937) se planteó la posibilidad de que la sepultura perteneciera a la actividad de los ocupantes del nivel B. La mayor parte de los investigadores (Bar-Yosef, 1998; Lieberman, 1993; Lieberman y Shea, 1994; Shea, 1998; Schwarcz et alii, 1998, etc) ha optado por esta última posibilidad, contemplando aTabun I dentro del estrato B. Sin embargo, algunos autores no aceptan esta correlación automática entre los neandertales y el Musteriense tipo B. Trinkaus (1993; Trinkaus y Ruff, 1999) ofrece argumentos para cuestionar este modelo, afirmando que el radio derecho $\mathrm{C} 4$, la diáfisis de fémur $\mathrm{C} 3$, al igual que el espécimenTabun C5 (todos ellos claramente insertos en el nivel C), forman parte del esqueleto de Tabun I. Por tanto, se barajan dos hipótesis; en primer lugar, que la atribución original de Tabun I al nivel Ces correcta, y que los huesos que representan C3, C4 y C5 se separaron del esqueleto antes de que el nivel B se depositara. Una segunda explicación supondría queTabun I pertenece al nivel B, o que es intrusivo en elC, pero pertenece a un momento posterior de ocupación.

En un artículo reciente, Schwarcz et alii (1998) ofrecen nuevas fechas por Series de Uranio para este esqueleto, con una media de $33.000 \pm 4.000$ BP. Una cronología tan moderna, en el caso de que aceptáramos la validez de estas nuevas analíticas (véase en contra Millard y Pike, 1999; Alperson $e t$ alii, 2000), implicaría que Tabun I fuera el espécimen neandertal más tardío del Próximo Oriente, en lugar del más antiguo como habíamos especulado. De ese modo, se plantearía la llegada de los neandertales a la región no antes del $70.0000 \mathrm{BP}$, como respuesta a los rigores del Würm europeo (Schwarcz et alii, 1998), y se podría acudir al modelo que correlaciona el Musteriense tipo $\mathrm{C}$ con los humanos modernos. A pesar de este problema y otros como la asignación taxonómica de algunos homínidos (Arensburg y Belfer-Cohen, 1998; Holliday, 2000; Wolpoff, 1989) o la cronología de los humanos modernos de Qafzeh y Skhul (p.e. Stringer, 1998), en el presente trabajo asumiremos la correlación entre los neandertales y el Musteriense tipo $\mathrm{B}$, y la de los humanos modernos con la facies C. Se trata de una elección funcional, ya que es así la única forma en la que podemos comparar, a través de la evidencia de los distintos yacimientos, el comportamiento de ambos tipos de homínidos. No obstante, quede patente que la asociación de una industria con un tipo de homínido determinado es una asunción en cierto modo artificial y no exenta de problemas.

\section{LOS ANÁLISIS ZOOARQUEOLÓGICOS}

En el Próximo Oriente, y siguiendo la influencia de los trabajos de Binford $(1981,1985)$, se ha asumido que los neandertales practicaron una estrategia mixta de caza y carroñeo que poco tenía que 
ver con la desarrollada tecnología cinegética de los humanos anatómicamente modernos (Bar-Yosef, 1989, 1994; Stiner, 1994; Stiner et alii, 1999, 2000, etc). Sin embargo, en nuestra opinión estas asunciones no tienen una base empírica sólida y se deben a ideas preconcebidas y a modas académicas al uso. Por desgracia, en el Próximo Oriente, como en muchas otras partes del continente, la zooarqueología ha sido utilizada sólo de forma complementaria y como simple comparsa a los análisis tecnotipológicos de la industria. Igualmente grave es que, en las contadas ocasiones en que han sido llevados a cabo los estudios faunísticos, éstos se han limitado a la identificación taxonómica y a los análisis de representación esquelética (Davis, 1977; Evins, 1982; Garrard 1982, 1983; Perkins, 1964; Takai, 1970; Wolf, 1988, etc). Sólo en los últimos años (Bar-Yosef et alii, 1992; Marean y Kim, 1998; Rabinovich, 1990, 1998; Rabinovich y Tchernov, 1995; Speth y Tchernov, 1998; Stiner y Tchernov, 1998; Stiner et alii, 1999, 2000) se han incorporado a los análisis zooarqueológicos tradicionales las disciplinas tafonómicas, como el estudio de las marcas de dientes y de corte, la atención a los procesos bioestratinómicos y diagenéticos, etc. Debido a estas deficiencias, los análisis comparativos entre neandertales y humanos modernos basados en la fauna se ven limitados por la escasez de información, siendo además ésta casi exclusivamente taxonómica. Con todo, los datos disponibles permiten llegar a algunas conclusiones.

A causa de la variabilidad ambiental y por tanto faunística que presenta el Próximo Oriente (véase Tchernov, 1992; Shea, 1998), tomaremos como ejemplo el área costera de Palestina. En esta zona, la cercanía entre yacimientos comoTabun, Kebara, Qafzeh, Skhul, etc, nos permiten asumir un mismo tipo de condiciones ecológicas, y por tanto podemos comparar la variabilidad en el registro faunístico de cada yacimiento. Como se puede observar en la tabla 2, las especies representadas en los distintos yacimientos no varían en demasía, bien sean asociados al Musteriense tipo C (Hayonim, Skhul y Qafzeh) o al tipo B (Kebara, Tabun B yAmud). En realidad, se trata de una tendencia lógica, ya que en un medio ambiente homogéneo (Skhul y Tabun, por ejemplo, están separados por unos pocos metros) es de esperar una representación de especies similar, siempre y cuando asumamos que las estrategias económicas durante ambas facies eran parecidas. Los porcentajes de representación taxonómica también reflejan un patrón semejante; la figura 1, basa-

\begin{tabular}{|c|c|c|c|c|c|c|}
\hline & \multicolumn{3}{|c|}{ Musteriense tipo $\mathrm{C}$} & \multicolumn{3}{|c|}{ Musteriense tipo B } \\
\hline & $\begin{array}{l}\text { HAYONIM } \\
\text { E Superior }\end{array}$ & SKHUL & $\begin{array}{c}\text { TABUN } \\
\mathrm{C}\end{array}$ & $\begin{array}{l}\text { KEBARA } \\
\text { VI-XII }\end{array}$ & $\begin{array}{c}\text { TABUN } \\
\text { B }\end{array}$ & AMUD \\
\hline Capreolus capreolus & + & $?$ & + & + & + & + \\
\hline Cervus elaphus & + & + & + & + & + & + \\
\hline Dama mesopotamica & + & + & + & + & + & + \\
\hline Sus scrofa & + & + & + & + & + & + \\
\hline Phacochoerus garrodae & $\ldots$ & + & $\ldots$ & $\ldots$ & $-\ldots$ & $\ldots$ \\
\hline Hippopotamus amphibius & $\ldots$ & -- & + & + & -- & $\ldots$ \\
\hline Gazella gazella & + & + & + & + & + & + \\
\hline Alcelaphus buselaphus & + & $?$ & + & + & + & + \\
\hline Capra aegagrus & + & + & + & + & + & + \\
\hline Bos primigenius & + & $t$ & + & + & + & + \\
\hline Equus sp. & + & + & + & + & + & + \\
\hline Dicerorhimus hemitoechus & + & $?$ & $?$ & + & $?$ & $?$ \\
\hline
\end{tabular}

Tab. 2. Herbívoros representados en yacimientos musterienses palestinos (a partir de los datos de Tchernov, 1992).

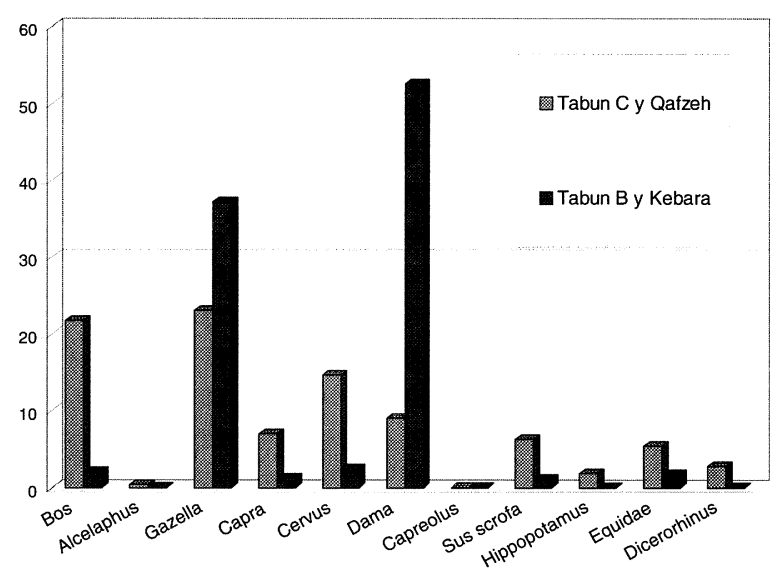

Fig. 1. porcentajes de representación anatómica de herbívoros en el Musteriense tipo C (ejemplos de Tabun C y Qafzeh XXII-V) y el tipo B (ejemplos de Tabun B y Kebara) (elaborado a partir de los datos de Bar-Yosef, 1989).

da en recuentos ya antiguos de especies (ver BarYosef, 1989), no permiten dilucidar una tendencia clara en la variación taxonómica, ni establecer diferencias concluyentes en la selección de especies en ambas facies del Musteriense levantino. Es cierto que, según este ejemplo, la incidencia en los grandes bóvidos (Bos primigenius) es mayor en el Musteriense tipo $\mathrm{C}$, mientras que en la fase $\mathrm{B}$ hubo un gran aprovechamiento de ungulados de menor tamaño (gacela en Kebara y gamo en Tabun B). Sin embargo, esta dicotomía no es extrapolable a otros yacimientos, ya que por ejemplo en los niveles del tipo C de Hayonim los ungulados de tamaño medio como Dama o Gazella representan entre el 79-93\% de las especies (Stiner y Tchernov, 1998), en Qafzeh el porcentaje de Gazella gazella es muy superior al que se pensaba (Rabinovich, 1998), mientras que por contra en yacimientos del Musteriense final comoAmud (Takai, 1970) queda también refle- 
jada la presencia de Bos, Equus y otros herbívoros de tamaño grande.

Asumiendo por tanto que las especies representadas y los porcentajes son similares tanto en los yacimientos asociados a humanos modernos como en los neandertales, cabe preguntarse cuál fue el medio de obtención de las carcasas. Como ya hemos señalado anteriormente, los estudios tafonómicos no son aún demasiado abundantes. Por ello, siguen predominando ideas preconcebidas como las de Bar-Yosef (1994: 25), quien afirma que "most Middle Paleolithic faunal assemblages reflect hunting of small and medium-sized animals and the opportunistic scavenging of large bovids. Upper Paleolithic animal bone assemblages were largely the results of hunting and trapping". Los animales de gran tamaño (uros, équidos e incluso rinocerontes) predominan en dos yacimientos musterienses al aire libre, Quneitra y Far'ah II. De este modo, la singular naturaleza de ambos asentamientos sirve a Bar-Yosef (1994) para asegurar que se trataba de una estrategia oportunista. Sin embargo, Gilead y Grison (1984) no hablan en ningún caso de carroñeo en Far'ah II, y los estudios originales (Davis et alii, 1988; Rabinovich, 1990) tampoco lo proponen para Quneitra. Bar-Yosef simplemente asume unos prejuicios muy extendidos sobre la incapacidad de los neandertales para cazar grandes animales, algo que sí se acepta para los humanos modernos. De este modo, incluso los propios colaboradores de Bar-Yosef, tras analizar la fauna de Kebara, no pueden sino concluir que "If the Kebara hominid is accepted as a Neandertal, then Neandertals... were already effective hunters, preferentially targeting large, and potentially dangerous, prime adult prey. Thus, at least in this one critical behavioral dimension there is nothing demostrably 'archaic' about the procurement practices of Levantine Neandertals" (Speth y Tchernov, 1998: 236).

Con todo, sí es cierto que existen algunas diferencias entre la fauna representada en el Musteriense con respecto al Paleolítico superior. Así, en el Próximo Oriente los grandes bóvidos y équidos que aparecen en el Paleolítico medio desaparecerán en el periodo posterior. Sin embargo, este cambio no está relacionado con la alternancia del carroñeo durante el Pleistoceno superior inicial, sino con una disminución gradual del número de animales de tamaño superior a los $250 \mathrm{kgs}$, quizás debida a los cambios climáticos y la intensificación de las actividades cinegéticas a causa de un aumento progresivo de la población humana (Davis et alii, 1988).
De hecho, estos autores señalan que en Quneitra los huesos aparecen muy completos, mientras que en yacimientos posteriores como Ein Gev I, la mayor parte de los restos óseos se encuentran totalmente fragmentados. De este modo, podría sugerirse que los grupos del Paleolítico superior y el Epipaleolítico explotaran en mayor grado las carcasas que los musterienses, aprovechando toda la carne e incluso la médula (Davis et alii, 1988). Si asumimos entonces que los humanos anatómicamente modernos consumían las carcasas en su totalidad, mientras que los neandertales (al menos los de Quneitra) abandonaban huesos completos todavía aprovechables, sería absurdo adscribir a estos últimos una estrategia carroñera.

En el Paleolítico superior seguirán estando representadas las mismas especies y en similares proporciones que en el periodo anterior, principalmente Gazella gazella y Dama mesopotamica (Lieberman, 1993; Rabinovich, 1998; Tchernov, 1992, etc). No obstante, algunos autores (Stiner y Tchernov, 1998; Stiner et alii, 1999, 2000) observan ciertos cambios entre las presas más pequeñas; en Hayonim, por ejemplo, la mayoría de los reptiles aparecen en el Musteriense (83\%) y los pájaros en el Paleolítico superior (92\%). De este modo, a partir del registro de Hayonim y de varios yacimientos musterienses italianos, Stiner et alii $(1999,2000)$ proponen que los reptiles serían más bien recolectados que cazados en el Musteriense, mientras que los avances tecnológicos del Paleolítico superior y Epipaleolítico permitirían a los grupos humanos obtener presas más móviles y rápidas como pájaros y lagomorfos. No obstante, tal afirmación debe ser matizada, ya que otros autores como Speth (BarYosef et alii, 1992) aseguran que la incidencia en presas pequeñas sólo es apreciable en el Paleolítico superior terminal, y no en los primeros tiempos del Ahmariense o Auriñaciense levantino. Con todo, y siguiendo los modelos propuestos por Klein $(1995,1998)$ para Sudáfrica, Stiner et alii $(1999$, 2000) aseguran que las mejoras tecnológicas del Paleolítico superior favorecerían el aumento demográfico y un mayor éxito cinegético que en el periodo anterior. Así, se aduce que los homínidos arcaicos no consiguieron un aumento demográfico porque no estaban capacitados tecnológicamente para ampliar su dieta.

Sin embargo, este proceso puede analizarse desde la perspectiva opuesta; si los recursos energéticos más ricos decrecen, tal y como demuestra la progresiva desaparición de la macrofauna en el 
Próximo Oriente, los grupos humanos se verían obligados a ampliar su espectro dietético, añadiendo recursos menos rentables y diversificando así sus alimentos. Es decir, que si animales como el auroch, los équidos o el hipopótamo desaparecen, mientras que se reduce el número de mamíferos de tamaño medio como el ciervo, el gamo o la gacela, es lógico que los humanos tuvieran que diversificar su dieta incorporando pequeñas presas como los pájaros y lagomorfos. Y es que, en realidad, tal y como señalan Marean y Assefa (1999), la captura de animales pequeños puede suponer un gasto energético muy alto y poco rentable. De este modo, sería absurdo pensar que los humanos modernos del Paleolítico superior capturaban pequeños animales de alta movilidad porque eran mejores cazadores; según esto, también podríamos afirmar que los neandertales no cazaban pájaros o lagomorfos porque su rica dieta no necesitaba recursos tan paupérrimos como los que proporciona la ingesta de un pequeño pájaro (Marean y Assefa, 1999).

En suma, parece que al menos para la zona del Mediterráneo oriental las diferencias en la fauna representada no son indicativas de unas estrategias económicas distintas entre humanos modernos y neandertales. Como venimos insistiendo, un problema básico a la hora de profundizar en el registro faunístico del Próximo Oriente es que, en general, sólo disponemos (y a veces ni siquiera eso) de recuentos taxonómicos, que aportan poca información sobre el comportamiento de los agentes que acumularon los restos óseos. No obstante, y a pesar de estos defectos, los análisis tafonómicos más novedosos también han sido desarrollados y aplicados al Musteriense del Oriente Próximo; hasta ahora, los estudios zooarqueológicos se basaban en el análisis y recuento de las epífisis y elementos anatómicos más fáciles de identificar. De este modo, los patrones de representación esquelética han estado siempre dominados por metápodos y elementos craneales. Tales secciones son escasas en contenido cárnico, por lo que se ha pensado que los humanos arcaicos practicaban una estrategia carroñera (Binford, 1981, 1985; Stiner, 1994).

Sin embargo, la aplicación en el yacimiento musteriense de Kobeh de un nuevo sistema analítico basado en la reconstrucción de elementos óseos a partir de secciones diafisarias, ha permitido elaborar un patrón de representación anatómica radicalmente distinto del obtenido por las técnicas tradicionales de análisis centradas en el estudio de los fragmentos de epífisis (Marean, 1998; Marean y
Kim, 1998; Marean y Assefa, 1999).Aplicando este modelo, los elementos representados son las secciones superiores del esqueleto (las más ricas en contenido cárnico), cuestionando así los modelos que abogaban por una estrategia carroñera. La aplicación de esta metodología en la cueva de Kobeh está sirviendo de acicate para su uso en otros yacimientos pleistocénicos, donde ahora se puede demostrar que los patrones de representación esquelética tradicionales son más un sesgo tafonómico que el resultado de la conducta antrópica. De este modo, podemos concluir que la evidencia disponible en el Próximo Oriente no permite adscribir a los neandertales una estrategia carroñera, por lo que parece claro que "The emphasis that lately has been given to the beginning of hunting $v s$. scavenging as related to a certain evolutional progress connected in Europe to the onset of the Cro-Magnon, is not relevant to the archeo-anthropological sequence in the Levant" (Rabinovich y Tchernov, 1995: 38).

\section{LOS MODELOS DE SUBSISTENCIA}

La reconstrucción de los modos de vida de los homínidos, la meta final de toda investigación, ha sido eludida por la mayor parte de los autores que trabajan en el Próximo Oriente. Salvo el antiguo intento de S. Binford (1968), tan sólo en los últimos años arqueólogos como Lieberman $(1993,1998)$ y Shea (1998; Lieberman y Shea, 1994) han elaborado hipótesis sobre los patrones de asentamiento en el área, intentando incluso dilucidar diferencias potenciales entre el comportamiento de ambos tipos de homínidos.

Como sabemos, los dientes de ciertos herbívoros reflejan una formación distinta de las capas de cemento de sus raíces, en función de la estación. Cuando los brotes gramíneos son tiernos, es decir, durante la época más húmeda (invierno), el proceso de masticación exige menos esfuerzo que en la época seca, cuando las hierbas son más duras y abrasivas. Esto queda reflejado en las capas de cemento de las raíces de los dientes mediante una orientación distinta de los cementoblastos. Si se observa la última capa, su tono y grosor nos indica el periodo estacional en el que falleció el animal. Lieberman (1993) ha aplicado este método a las piezas dentarias de Gazella gazella de los yacimientos musterienses de Kebara (unidades VII-X), Qafzeh (VIIXXIII), Hayonim E y en los niveles D, C y B de Tabun. Según sus análisis, en Kebara y Tabun B 
parece que las presas fueron cazadas durante todo el año: en Kebara, el 52\% de las gacelas fallecieron en primavera/verano, y el $48 \%$ durante el otoño invierno, tendencia que también se repite en Tabun B. Por el contrario, en Qafzeh XVI-XXIII, Hayonim E y Tabun C-D el patrón que se desprende apunta a una ocupación estacional, ya que o bien los animales fueron cazados en otoño/invierno (caso de Tabun C-D), en primavera (Hayonim E) o en primavera/verano, tal y como ocurre en los niveles XVIXXIII de Qafzeh. En suma, Lieberman (1993; Lieberman y Shea, 1994), generalizando estos ejemplos al resto del área palestina, sugiere que los neandertales (Kebara y Tabun nivel B) mantenían una estrategia de asentamiento poco móvil (patrón multiestacional o radial), mientras que los humanos modernos (en base a la evidencia de Qafzeh y Tabun C) ocuparon el territorio en un modelo más migratorio (patrón circular).

John Shea $(1988,1989)$ ha estudiado los útiles apuntados de los yacimientos de Kebara (unidades IX-XII), Tabun B, Qafzeh (niveles XVII-XXIV y $\mathrm{XV}$ ) y Tabun nivel C. Según este autor, las huellas de desconchados, estriaciones y pulidos sugieren que estos artefactos fueron utilizados como puntas enmangadas. Además, se identifican huellas de impacto, lo que indica que también sirvieron como proyectiles. Estudiando las trazas de uso en los citados yacimientos, Shea observó que los porcentajes más elevados de microhuellas de impacto se daban en Kebara y Tabun B, precisamente donde el número de puntas era mayor. De este modo, y asumiendo siempre el papel cinegético de las puntas, Shea concluye que los neandertales hacían mayor incidencia en la caza que los humanos modernos. Este modelo teórico será más tarde (Shea, 1998) ampliado y desarrollado, como en seguida veremos.

Los innovadores análisis de ambos autores quedaron reflejados en un trabajo conjunto (Lieberman y Shea, 1994), que confluía hacia la idea de que neandertales y humanos modernos, al menos en el caso de la zona palestina meridional, hicieron un uso diferente del espacio y los recursos del medio. Así, y como ya hemos señalado, se propuso que los humanos modernos adoptarían un patrón de asentamiento circular. Esta estrategia supone un movimiento estacional continuo de un campamento efímero a otro, en un ciclo anual recurrente (Binford, 1980). Por el contrario, el modelo radial implica la existencia de uno o varios campamentos base residenciales y multiestacionales, desde los que se organizaría un movimiento radial a partir de campa- mentos efímeros cercanos, dando lugar a lo que Binford (1980, 1983) denomina una estrategia logística. Para Lieberman y Shea (1994), y en un medio como el de Palestina, es muy posible que los colectores de un modelo radial incurrieran en mayores costes energéticos diarios que los forrajeros adscritos a estrategias circulares, ya que haciendo un uso restringido del espacio, se enfrentarían a un potencial agotamiento de los recursos, y al peligro de las variaciones estacionales en la distribución de los mismos. De ese modo, y según Lieberman, paradójicamente el modelo circular debió finalmente requerir menos movilidad que la estrategia radial, ya que los grupos conseguirían suficientes recursos de alta calidad en medios no agotados, trasladándose a nuevos asentamientos cuando fuera necesario. Así, y dando un último paso argumental, se propone que las estrategias de asentamiento de los neandertales (modelo logístico) no pudieron competir a largo plazo con el mejor modelo adaptativo de los humános modernos, basado en una alta movilidad residencial, siendo quizás ésta una de las causas principales de la extinción de los primeros (Lieberman y Shea, 1994).

A pesar del interés de las reconstrucciones conductuales de Shea y Lieberman, su modelo presenta una serie de problemas graves, tanto argumentales como metodológicos. En cuanto a estos últimos, hay que comenzar señalando que para establecer un análisis de estacionalidad sobre grupos humanos es preciso estar en disposición de descartar otros agentes en los procesos de configuración de los conjuntos óseos de los yacimientos arqueológicos y poder atribuir así su autoría a la acción exclusivamente antrópica. Para ello, es necesario realizar estudios tafonómicos completos, de los que, salvo algunas excepciones ya mencionadas, en la actualidad la investigación paleolítica del Próximo Oriente se encuentra exenta. Por otro lado, definir la estacionalidad de un yacimiento exclusivamente a partir de los estudios de los cementoblastos es muy arriesgado, y habría que tener también en cuenta la propia naturaleza del depósito, su profundidad, la actividad diagenética, la densidad de artefactos, la diversidad de útiles, etc. Además, y tal y como señala Olszewski (1993), hablar de suelos prístinos en depósitos kársticos es casi una utopía, encontrándonos la mayor parte de las veces ante palimpsestos que pueden reflejar una sucesión de ocupaciones de cientos de años. De este modo, es difícil aplicar criterios de estacionalidad a conjuntos como Tabun C, B o Qafzeh, donde en ningún caso encon- 
tramos suelos de ocupación con una temporalidad bien delimitada.

Como ya hemos señalado, la identificación automática que se hace entre las facies y los tipos humanos no es aceptada por todos los autores (p.e. Trinkaus, 1993; Arensburg y Belfer-Cohen, 1998, etc). En cualquier caso, y aceptando esta premisa, las deducciones de Lieberman y Shea (1994) también resultan equívocas; en los niveles XV-VII de Qafzeh, Lieberman (1993) descubre un patrón de ocupación multiestacional, y Shea (Lieberman y Shea, 1994) obtiene un gran número de puntas, muchas de ellas con huellas de impacto. De este modo, ambas metodologías confluyen en una ocupación radial con gran incidencia en la caza, relacionando estos niveles de Qafzeh con los de Tabun B y Kebara, y por tanto reforzando su teoría sobre la economía de los neandertales levantinos. Pues bien, lo cierto es que la mayor parte de los autores rechazan desde hace años que en Qafzeh existan diacrónicamente el Musteriense tipo C y el tipo B, adscribiéndose en la actualidad todo el depósito a la fase C (Bar-Yosef, 1998; Boutié, 1989; BelferCohen, 1993; Goren-Inbar y Belfer-Cohen, 1998, etc). Por todo ello, nos encontraríamos ante un patrón multiestacional y con gran incidencia cinegética adscrito a los humanos modernos, y no a los neandertales, cuestionando así el modelo dicotómico de Lieberman y Shea (1994). Un problema similar presenta Hayonim nivel E; Lieberman y Shea no encuentran diferencias internas en este depósito, cuando hoy día parece claro que se distinguen dos facies distintas, el Musteriense tipo D y el tipo C (Bar-Yosef, 1998; Meignen, 1998; Goldberg y BarYosef, 1998), por lo que se estaría mal interpretando su relación con el resto de los ejemplos. También podemos presentar una objeción con respecto al problema del Musteriense tipo D; Lieberman y Shea (1994) incluyen Tabun nivel D y Hayonim E (recordemos que parte de este nivel pertenece a la facies tipo D) en el modelo circular de Tabun nivel C y Qafzeh. Según el razonamiento de los autores, la estrategia radial de los neandertales está relacionada con un mayor énfasis en la caza, tal y como demuestra el elevado número de puntas. Entonces ¿cómo se relaciona el Musteriense tipo D, la facies más rica en puntas, con una estrategia circular poco especializada en las actividades cinegéticas?.

Como vemos, tanto la metodología como las premisas que van proponiendo Lieberman y Shea carecen de una base sólida. Con todo, y aunque aceptáramos su hipótesis sobre la diferenciación entre dos estrategias económicas, las conclusiones generales que los autores extraen a partir del ejemplo palestino son ya muy difíciles de asumir. Así, aseguran que "the more-circulating pattern of residential mobility typical of modern humans is more energetically efficient than the more-radiating mobility pattern that characterized the archaic humans of the Levant" (Lieberman y Shea, 1994: 320). De este modo, los autores obvian que los sistemas radiales y circulares pueden ser compatibles y no excluyentes (Binford, 1980, 1983). Además, tal y como señala Belfer-Cohen (1993), un modelo radial o logístico es mucho más complejo que uno circular, requiriendo mayor planificación, por lo que la supuesta superioridad energética de este último modelo es más bien dudosa. Lieberman y Shea basan su categorización de los patrones de asentamiento en los trabajos de Binford (1980, 1983). Por esta razón, no entendemos por qué Lieberman asegura el mayor éxito económico de las estrategias circulares, cuando el propio Binford (1980: 18) llega a afirmar que "under the same conditions increased logistical dependence with an accompanying reduction in residential mobility would be favored".

Podemos dar el siguiente paso teórico, aceptando la metodología de Lieberman y Shea, errónea en varios de sus puntos, $\mathrm{y}$ dando por válidas las deducciones de los autores, aún más discutibles. Así, podríamos asumir que los neandertales de la región de Palestina practicaron una estrategia económica logística, los humanos modernos un patrón de asentamiento circular, y que este último fue finalmente más rentable energéticamente que el adoptado por los neandertales de la zona. Sin embargo, en ningún caso podemos aceptar que este ejemplo particular con tan sólo cuatro cuevas palestinas sirva para afirmar que la estrategia radial practicada por los neandertales les abocó a su desaparición en toda Europa. Esto supone extrapolar un caso concreto (bastante problemático por otra parte) al resto del registro euroasiático. De hecho, su modelo no es operativo ni siquiera para la región del Próximo Oriente, tal y como demuestra el ejemplo del área de Hisma. En esta zona, Henry $(1992,1995)$ propuso un modelo circular estacional para los asentamientos de Tor Faraj y Tor Sabiha. Sin embargo, y pese a que en todos su trabajos anteriores este autor relacionaba estos conjuntos con el Musteriense tipo D, en la actualidad (Henry, 1998) los adscribe el tipo B. Por tanto, si asumiéramos, tal y como hacen Lieberman (1993; Lieberman y Shea, 1994) 
y Shea $(1995,1998)$, que el Musteriense tipo Tabun $\mathrm{B}$ se asocia con los neandertales, entonces en el sur de Jordania tendríamos a estos homínidos ocupando el territorio de una manera circular y estacional, supuestamente más rentable energéticamente. De este modo, insistimos, queda patente que el modelo propuesto por Lieberman y Shea (1994), no debe generalizarse ni siquiera a la totalidad del Próximo Oriente, y no ha de considerarse como una explicación plausible de las diferencias conductuales entre humanos modernos y neandertales, y mucho menos como paradigma explicativo de la extinción de estos últimos.

La nueva hipótesis de Shea $(1995,1998)$ es sugestiva, y además tiene el mérito de intentar poner en relación los yacimientos del área continental (zona de estepa) con los de la costa (área boscosa). En la región marítima, la fauna que predomina en los yacimientos es la de tamaño medio, como jabalí, ciervo, gamo y gacela. La mayoría de estos animales son solitarios o viven en pequeños grupos dispersos, lo que haría difícil a los cazadores predecir su ubicación. Según Shea (1998), centrarse en la caza selectiva de alguna de estas especies sería muy costoso energéticamente, por lo que lo más rentable sería un comportamiento cinegético oportunista, que incluyera un amplio espectro de especies. Siguiendo con el razonamiento, el cazador podría rentabilizar el tiempo de búsqueda recolectando vegetales. Por ello, para Shea las armas de caza usadas en las tierras boscosas deberían ser versátiles, por lo que lo más recomendable serían las lanzas con punta de madera, fáciles de reavivar o de remplazar, y no las de sílex, pues la obtención, manufactura y reparación de estas últimas robaría tiempo que podría dedicarse a la recolección de alimentos. Por el contrario, en la estepa irano-turania, las plantas comestibles se encuentran muy dispersas, por lo que los animales deben migrar continuamente buscando los recursos vegetales. $\mathrm{La}$ mayoría de estos animales, en contra de los de bosque, son gregarios y suelen migrar en un ciclo estacional predecible.

De este modo, para Shea lo más rentable en el área esteparia sería la caza de acecho o de interceptación, ya que los homínidos conocerían perfectamente los movimientos migratorios de los animales. Esta caza se llevaría a cabo en puntos concretos del paisaje, en los que los cazadores acumularían piezas líticas, mucho más eficientes además contra animales como el dromedario, el caballo y onagra, precisamente los más abundantes en el registro del
Musteriense continental. Como ya hemos visto, algunas puntas Levallois presentan huellas de impacto indicativas de que fueron utilizadas como proyectiles (Shea, 1988, 1989, 1995, etc). Por ello, y en vista de lo anterior, sería lógico que hubiera un porcentaje más elevado de puntas en la región esteparia, allí donde en teoría las actividades cinegéticas estarían más desarrolladas. Partiendo de esta hipótesis, Shea (1998) ha comparado los porcentajes de puntas con respecto al número de núcleos en gran parte del registro musteriense del Próximo Oriente, coincidiendo los índices con los resultados esperados; en la zona septentrional costera, la región más húmeda y boscosa, los porcentajes de puntas son inferiores a los de la costa israelí y sensiblemente más bajos que en el área esteparia. De este modo, en función del mayor número de puntas de proyectil de los yacimientos del interior y de la poca variedad de especies representadas en esta región, para Shea parece obvio que fue la caza organizada y en emboscada lo que predominó en la zona árida, frente a las estrategias más expeditivas del medio costero. Este autor da luego otro paso teórico, e intenta diferenciar, como ya hizo en trabajos anteriores (Lieberman y Shea, 1994; Shea, 1995), el comportamiento de humanos modernos y neandertales. Asumiendo que el Musteriense tipo $\mathrm{C}$ se asocia a los modernos y el tipo Tabun $\mathrm{B}$ a los neandertales, Shea (1998) compara los porcentajes de puntas de unos y otros depósitos. Como era de esperar, el tipo B presenta un mayor número de puntas que el Musteriense tipo C. Por fin, y concluyendo su razonamiento, sería evidente que los neandertales practicaron una caza de interceptación, basando la mayor parte de su dieta en las proteínas animales, mientras que los humanos modernos, con estrategias cinegéticas más oportunistas, ampliarían el abanico de recursos potenciales haciendo una mayor incidencia en los alimentos vegetales.

El estudio de Shea (1998), como los anteriores (Lieberman y Shea, 1993; Shea, 1995), presenta graves deficiencias argumentales y metodológicas. Este autor basa toda su hipótesis en la consideración de que las puntas Levallois sirvieron principalmente como proyectiles (Shea, 1988, 1989, 1995, etc). A partir de esta asunción, elabora una teoría interpretativa sobre las diferentes estrategias de asentamiento en la región. Pues bien, ya desde el comienzo los argumentos de Shea son muy discutibles; los nuevos estudios de Plisson y Beyries (1998) demuestran que las huellas de impacto en las puntas de Kebara, uno de los yacimientos en los que Shea

T. P., 58, n. ${ }^{\circ} 1,2001$ 
fundamenta su teoría, no son en absoluto tan numerosas como este autor pretendía.Además, estudiando otros yacimientos como Umm el Tlel, y desarrollando un extenso programa experimental, se ha llegado a la conclusión de que los criterios de Shea para considerar las puntas Levallois como proyectiles son en gran medida erróneos, encontrándose huellas traceológicas que indican una gran diversidad funcional de las puntas Levallois (trabajo de la madera, despiece de carcasas, etc), por lo que no puede asumirse una única utilidad para estos artefactos (Plisson y Beyries, 1998). Cuestionando la asunción de Shea $(1995,1998)$ de que el porcentaje de puntas Levallois es un reflejo de la incidencia cinegética de cada yacimiento, toda su teoría interpretativa queda invalidada. No obstante, y a pesar de ser errónea, podríamos asumir esta hipótesis y ver si el resto de los postulados de Shea (1998) son correctos. Por tanto, aceptemos provisionalmente que las puntas Levallois en cada yacimiento son indicativas de las actividades cinegéticas.

Sin embargo, los siguientes pasos del razonamiento también son muy cuestionables; la explicación que Shea propone sobre el ahorro energético en la elaboración de útiles con respecto a los grupos humanos de bosque no es muy creíble. Tal y como señala Kuhn (1998), se podría plantear la hipótesis al revés; siendo la caza oportunista más impredecible, los grupos que practicaran este tipo de estrategia deberían minimizar los riesgos de perder la presa facturando buenas armas de caza. Por el contrario, los grupos que adoptaran una actividad cinegética de interceptación de las manadas, gozarían de un mayor número de oportunidades para conseguir carne, por lo que en este caso "the absolute effectiveness of weapons is less crucial" (Kuhn, 1998: 67). Otro de los problemas fundamentales del modelo es que el autor establece los porcentajes de puntas en los yacimientos comparándolos con el número de núcleos (Shea, 1998), en lugar de compararlos con el total de los productos Levallois (Shea, 1995). Esto presupone que todos los yacimientos fueron campamentos base a los que se aportaron nódulos, facturando los útiles in situ. En muchos de los casos, no tendría por qué ser esa la naturaleza del asentamiento, y bien podría tratarse de puntos a los que se trasladaron productos de talla finalizados, alterando así los porcentajes. Un ejemplo significativo es el de Tor Sabiha, precisamente en el que Shea (1998) encuentra el índice más alto de puntas Levallois. Indagando en la publicación original de este yacimiento (Henry, 1995), observamos que es considerado un campamento efímero en el que el porcentaje de útiles convergentes con respecto al número de núcleos es tan elevado simplemente porque las puntas Levallois fueron manufacturadas previamente a su inclusión en el yacimiento, y no porque se observe una especial actividad cinegética. Shea también obvia la distancia a las fuentes de aprovisionamiento de materia prima, presuponiendo que la lejanía o proximidad del sílex no influye en el porcentaje de productos manufacturados, asunción que muchos autores (Hovers, 1998; Henry, 1998b; Kuhn, 1998) consideran errónea. Por tanto, la naturaleza del yacimiento, la distancia a las materias primas, así como la cantidad del depósito excavado, la variabilidad del conjunto, la distribución microespacial, etc, afectan de manera significativa a la abundancia de puntas que aparecen en cada yacimiento y hacen más que cuestionable la elaboración de porcentajes a partir de su comparación con el número de núcleos.

Pese a todos estos problemas, que ya de por sí harían poco fiable cualquier tipo de análisis estadístico utilizando estas variables, podemos analizar los resultados obtenidos por Shea (1998). Según este autor, el porcentaje de puntas en la costa septentrional es de $0.44,0.59$ para la misma latitud en el interior, 1.61 en la costa sur y 3.67 en el interior me-

\begin{tabular}{|l|l|l|l|l|}
\cline { 2 - 5 } \multicolumn{1}{c|}{} & \multicolumn{4}{c|}{ Musteriense } \\
\cline { 2 - 5 } \multicolumn{1}{c|}{} & Tipo D & Tipo C & Tipo B & Tipo B/C \\
\hline Costa norte & $(3) 1.29$ & $(4) 0.07$ & $(3) 0.26$ & $(0) 0$ \\
\hline Interior norte & $(7) 0.7$ & $(0) 0$ & $(0) 0$ & $(7) 0.63$ \\
\hline Costa sur & $(1) 1.97$ & $(5) 0.33$ & $(7) 2.35$ & $(4) 0.61$ \\
\hline \multirow{2}{*}{ Interior sur } & $(9) 3.72$ & $(0) 0$ & $(0) 0$ & $(0) 0$ \\
\cline { 2 - 5 } & $(4) 0.89^{*}$ & $(0) 0$ & $(2) 3.25^{*}$ & (0) 0 \\
\hline
\end{tabular}

Tab. 3. Relación puntas Levallois/núcleos según la región. Los porcentajes se han obtenido a partir de los datos de Shea (1998). (NOTA 1).

NOTA 1: ( ) número de yacimientos-niveles incluidos en la muestra. * Se modifican los datos en la zona del interior meridional, adscribiendo Tor Faraj y Tor Sabiha al Musteriense tipo B (Henry, 1998), y corrigiendo los porcentajes erróneos de puntas Levallois/núcleos ofrecidos por Shea para Tor Faraj, en realidad con una media de 1.20 (Henry, 1998b). El nivel 10 de Yabrud I, considerado por Nishiaki (1998) como perteneciente al tipo D, no se incorpora a nuestro estudio, pues Shea no se pronuncia sobre su adscripción a una facies determinada. Igual ocurre con Geula, Kebara VII-VIII y Quneitra, presentes en el estudio original de Shea (1998). 


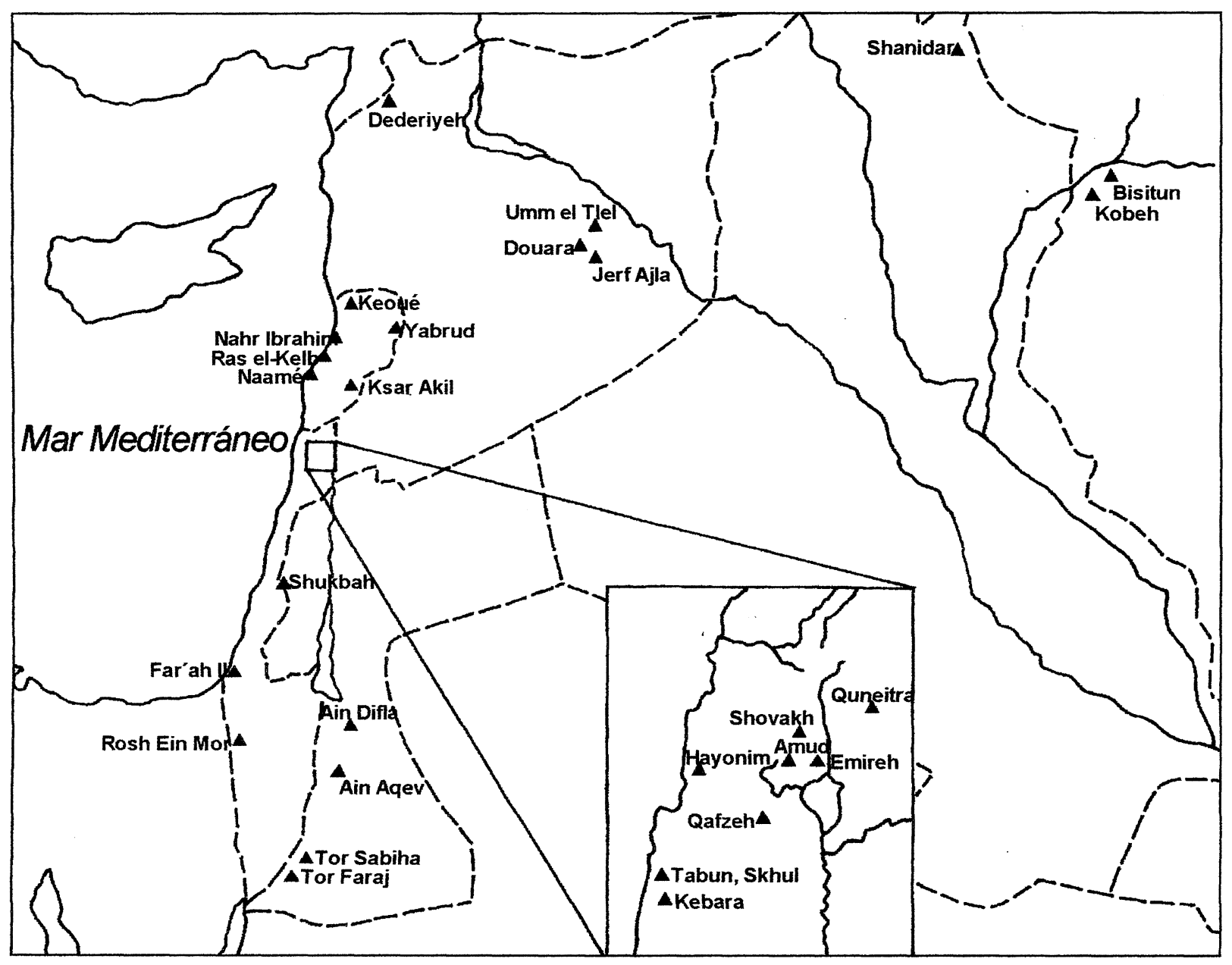

Fig. 2. Yacimientos musterienses del Próximo Oriente mencionados en el texto.

ridional. A pesar de que las diferencias regionales no son significativas a nivel estadístico, sí es cierto que los índices de puntas/núcleos irían aumentando en función de la aridez, tal y como se proponía en el modelo teórico. Sin embargo, Shea incurre en un nuevo error, ya que obtiene los porcentajes en cada región mezclando los yacimientos de distintas facies, por tanto de muy diferentes cronologías y, según convienen los distintos autores, de distintas especies de homínidos. No aceptando estos resultados artificiales, hemos elaborado un análisis usando los porcentajes ofrecidos por Shea de cada yacimiento, pero separándolos por región y por facies. Observando nuestros resultados (Tab. 3), se aprecia que no existe un incremento progresivo de los porcentajes de puntas Levallois según aumenta la aridez, máxime si incorporamos Tor Faraj y Tor Sabiha al Musteriense tipo B, tal y como se consideran en la actualidad (Bar-Yosef, 1998; Henry, 1998).
Además, si retiramos Tor Sabiha de la muestra, por las razones antes expuestas, el porcentaje resultante para el interior meridional es de 1.20, inferior entonces al de la costa meridional. En suma, lo que nos están indicando nuestros datos es que, separando los yacimientos por periodos, no hay ninguna correlación entre la latitud y los porcentajes de puntas. La explicación de los resultados de Shea es sencilla; mezclando yacimientos del tipo D y B, siempre muy ricos en puntas Levallois, con los del tipo C, con escasas puntas Levallois, los porcentajes dependían del número de yacimientos de cada facies en las distintas regiones. Si los del tipo D eran los más numerosos (tal y como Shea pretendía para el Levante interior meridional), la media resultante obviaría la presencia de uno o dos yacimientos del tipo $\mathrm{C}$, ocurriendo lo contrario para las zonas septentrionales. La propia posición geográfica de los distintos yacimientos (Fig. 2) nos lleva a una 
nueva objeción ante la hipótesis de Shea. Si el Musteriense tipo B está relacionado con estrategias cinegéticas de interceptación, típicas del medio árido, no tiene sentido encontrar yacimientos de esta facies como Keoué, Amud, Kebara, Tabun 117, en zonas costeras húmedas.Además, depósitos como los de Tabun (Jelinek, 1981, 1982) o Yabrud I (Nishiaki, 1998) muestran la sucesión de las tres facies (D, C y B) en un mismo yacimiento, siendo entonces difícil sostener que las condiciones ecológicas variaron radicalmente de un periodo a otro, o que los grupos del Musteriense tipo B usaban estrategias de caza de manadas en lugares donde sólo disponían de animales no gregarios.

El último paso teórico de Shea (1998) es la búsqueda de diferencias conductuales entre neandertales y humanos modernos. Así, concluye que los neandertales cazaban más que los Homo sapiens sapiens, y además practicando estrategias de acecho a manadas. Esto último ya lo hemos discutido, y parece claro que Shea no tiene una base sólida en la que sustentarse. Con respecto a lo primero, el razonamiento de Shea podría resultar válido; si las puntas Levallois sirvieron para cazar (pese a que esto no es estrictamente cierto, como ya hemos visto), y en los conjuntos del tipo B son mucho más numerosas, todo ello implica que los neandertales hicieron una mayor incidencia en los recursos cárnicos que los humanos anatómicamente modernos. Volvemos al mismo problema, la dificultad de considerar a un útil, en este caso la punta Levallois, como reflejo de una estrategia económica durante un periodo de tiempo tan amplio. Como sabemos, el nivel $\mathrm{C}$ de Tabun está hoy datado en torno a los 170.000 años (Mercier et alii, 1995). Pensar que las puntas Levallois que encontramos en ese depósito pueden medir la intensidad cinegética de los grupos humanos comparando sus porcentajes con los obtenidos por ejemplo en Amud, 100.000 años más moderno (Valladas et alii, 1999) es cuando menos arriesgado. Pero además, es un problema conceptual; la explicación que propone Shea (1998) para el uso de las puntas es excesivamente funcionalista, dando a la variabilidad de los conjuntos industriales una única dimensión. De este modo, "Levallois points cannot be seen as a morphological type with any necessary functional correlate, such interpretation of activities based on the proportional occurrences of Levallois points are without empirical foundation" (Marks, 1998: 20). Por todos estos problemas, parece claro que las hipótesis de Shea (1998) no pueden servir como referencia para distinguir modelos subsistenciales durante el Paleolítico medio en el Próximo Oriente, y por ende diferentes marcos conductuales entre neandertales y humanos modernos.

\section{LOS HOMÍNIDOS}

La muestra de neandertales del Próximo Oriente procede de las cuevas de Tabun, Kebara, Amud, Dederiyeh, y Shanidar, todas ellas con esqueletos completos o parcialmente completos, a los que se pueden sumar los restos aislados de Shovakh. Por su parte, los restos óseos de humanos anatómicamente modernos en contextos del Pleistoceno superior inicial se restringen a los hallazgos de Qafzeh y Skhul. Yacimientos como Bisitun, Ksar Akil, Hayonim y Shukbah han proporcionado restos aislados, pero por su carácter fragmentario no pueden adscribirse a una especie en concreto. Los individuos de Qafzeh y Skhul se caracterizan por un mentón prominente, un espacio retromolar reducido o ausente, una fosa canina desarrollada, una cavidad nasal pequeña, órbitas oculares altas, un torus supraorbital reducido o inexistente, y una calota craneal redondeada (Mann, 1995). Por otro lado, el grupo neandertal representado por Shanidar, Amud, Tabun I, Kebara 2 y los individuos de Dederiyeh, se caracterizan por una calota craneal más alargada, un torus supraorbital prominente, un área retromolar más obvio y asociado a una cara más proyectada, a menudo con un gran maxilar y un escaso o inexistente mentón.

La impresión inicial al estudiar la muestra de Qafzeh y Skhul es que las poblaciones humanas modernas son menos robustas que las neandertales. Esto se observa en los índices de robustez articular y en el diámetro diafisario de los huesos de las extremidades. También se observa en los humanos de Qafzeh y Skhul con respecto a los neandertales otras diferencias, plasmadas en una anchura menor de sus escápulas, en la dimensión reducida de los túneles carpales, las tuberosidades distales de las falanges de la mano, la reducción del quadriceps femoris y en otros rasgos adicionales. Por ejemplo, los fémures tienen un cuello largo y las diáfisis tienden a mostrar pilastres. Por otro lado, los índices crurales se encuentran entre los más elevados de cuantos se conocen en nuestra especie en la actualidad. En conjunto, todos estos rasgos en el esqueleto postcraneal apuntan a una menor robustez, aunque ésta es considerablemente mayor en ambos grupos de homíni- 
dos de la que se observa en periodos posteriores en Homo sapiens sapiens. Basándose en todas estas características, Trinkaus $(1989,1992)$ ha intentado encontrar posibles diferencias conductuales entre ambos tipos de homínidos a través de la interpretación funcional de la morfología de los restos óseos. Según sus estudios, la reducción de la musculatura de los miembros superiores, del tamaño de la escápula y de los carpales y falanges, etc, indica que los humanos modernos de Skhul y Qafzeh gozaban de una mayor precisión en el uso de la mano y en la movilidad del brazo (ver también Niewoehner, 2001). Por sí solo, sin embargo, este dato no ofrece ningún tipo de inferencia en las estrategias económicas y de asentamiento de ambos tipos de homínidos. Otro de los argumentos de Trinkaus (1989, 1992) es la hipertrofia cortical de los huesos y la gran musculatura postcraneal de los neandertales levantinos, lo que según este autor sugiere que estos homínidos destinaban gran parte de su tiempo a la deambulación por el paisaje, más del que se infiere a partir de los restos apendiculares de Qafzeh y Skhul. Esta inferencia sí supone un argumento a favor de la existencia de diferencias conductuales entre modernos y neandertales. Sin embargo, y tras el reciente estudio del grosor y geometría de las secciones de los húmeros, fémures y tibias, el propio Trinkaus (1995; Trinkaus y Ruff, 1999, 1999b; Trinkauset alii, 1998) afirma no encontrar diferencias significativas en la robustez de los miembros inferiores de neandertales y modernos levantinos, por lo que reconoce que ambos tipos de homínidos estuvieron sujetos a similares procesos de estrés en sus patrones de movilidad por el territorio.

De igual modo, Lieberman (1998), que años antes (Lieberman, 1993) había llegado a sugerir que los energéticamente costosos patrones de asentamiento neandertales habían contribuido a su extinción, ha cambiado ahora su opinión. Estudiando el grado de crecimiento de las corticales de los huesos, este autor ha observado que los miembros apendiculares inferiores de los humanos modernos y de los neandertales del Próximo Oriente eran muy similares en su robustez, "suggesting that both taxa engendered higher levels of mechanical loading from walking or running in comparison with recent modern humans" (Lieberman, 1998: 268), de modo que aunque "Neandertals and modern humans may have used somewhat different mobility strategies, the energetic costs of their mobility patterns appear to have been roughly equivalent" (Lieberman, 1998: 70).
La última de las diferencias que Trinkaus (1989, 1992) observaba en la muestra de ambas especies era el elevado número de marcas en los dientes de los neandertales levantinos, que no se encuentra en los individuos de Qafzeh y Skhul. Este patrón de estrías transversales está probablemente relacionado con un uso paramasticatorio de la dentición en los neandertales. Con todo, el uso paramasticatorio no puede servir como argumento para afirmar, tal y como hizo Trinkaus (1992), que los neandertales carecieron de la tecnología de los humanos modernos de Skhul y Qafzeh, pues estos últimos no necesitaron de los dientes para llevar a cabo sus tareas. Se trata de una proposición ciertamente arriesgada, ya que los materiales arqueológicos de Qafzeh y Skhul en absoluto parecen superiores tecnológicamente a los asociados a sus vecinos neandertales. Además, numerosos estudios tienen perfectamente documentados los casos de uso paramasticatorio en sociedades cazadoras-recolectoras actuales como los aborígenes australianos (véase Brown y Molnar, 1990; Molnar et alii, 1989, etc), que estamos seguros Trinkaus no querría categorizar como "tecnológicamente inferiores" a los humanos modernos del Paleolítico medio. Vemos por tanto que, hasta el momento, el estudio paleoantropológico de los restos levantinos tampoco parece ofrecernos argumentos convincentes para elaborar patrones dicotómicos sobre las estrategias económicas de los humanos modernos y neandertales del Pleistoceno superior inicial. Por todo ello, quizás "the real behavioral constrasts may have been social and organizational, and not directly linked to the technology of food acquisition and processing" (Trinkaus $e t$ alii, 1998: 403). Veamos ahora si encontramos las diferencias sociales que buscan Trinkaus y sus colaboradores. En el Próximo Oriente disponemos de indicios directos sobre los aspectos sociales y simbólicos de ambos grupos de homínidos: las sepulturas.

\section{LOS ENTERRAMIENTOS}

La zona del Próximo Oriente ha sido especialmente generosa en restos humanos interpretados como sepulturas. De este modo, se puede distinguir entre el grupo de Tabun, Kebara, Dederiyeh, Amud y Shanidar, todos ellos neandertales, y el complejo representado por las sepulturas de Skhul y Qafzeh, las únicas conocidas de humanos modernos en el Paleolítico medio euroasiático, ya que la preten-

T. P., 58, n. $^{\circ} 1,2001$ 
dida inhumación musteriense de Staroselje (Crimea), ha resultado ser un enterramiento medieval (ver Monigal et alii, 1997).

El número de sepulturas en el Próximo Oriente varía en función de la interpretación de cada autor. Autores como Gargett $(1989,1999,2000)$ niegan cualquier tipo de evidencia funeraria en el Paleolítico medio, buscando explicaciones alternativas basadas en los procesos tafonómicos y la conservación diferencial de los restos. En realidad, los análisis de Gargett $(1989,1999,2000)$ no ofrecen ni un solo argumento consistente para negar la existencia de las sepulturas musterienses. No debemos sin embargo restar relevancia a sus trabajos, ya que ciertamente suponen una llamada a la reflexión y a la profundización en los procesos de formación de los yacimientos, como etapa previa a la elaboración de hipótesis a veces demasiado arriesgadas. Con todo, lo cierto es que la mayoría de los autores aceptan la existencia de sepulturas musterienses en el Próximo Oriente. Así, para Kebara se habla de uno (Tillieret alii, 1988, 1991) o dos enterramientos (Hovers et alii, 1995; Belfer-Cohen y Hovers, 1992; Defleur, 1993), dos para Dederiyeh (Akazawa et alii, 1995; Akazawa et alii, 1999) uno para Tabun (Belfer-Cohen y Hovers, 1992; Hovers et alii, 1995; Defleur, 1993) y tres para Amud (Hovers et alii, 1995, 2000). Más controvertido es el caso de Shanidar, donde Defleur habla de hasta seis sepulturas (individuos IV,VII) y otros sólo de dos, Shanidar I y IV (BelferCohen y Hovers, 1992; Hovers et alii, 1995). Semejantes problemas presentan los restos de humanos modernos, ya que en Skhul varía el número de sepulturas propuestas entre los cuatro (Tillieret alii, 1988; 1991), seis (Defleur, 1993; Belfer-Cohen y Hovers, 1992) y siete individuos (Hovers et alii, 1995), y en Qafzeh entre los seis (Defleur, 1993; Hovers et alii, 1995; Tillier et alii, 1988; 1991) y siete (Belfer-Cohen y Hovers, 1992) enterramientos. No es nuestro objetivo aquí discernir los matices que sopesa cada investigador, pero lo cierto es que, desde un término medio, podemos asumir que un importante número de individuos identificados en casi todas las cuevas fueron objeto de un enterramiento intencional.Veamos entonces cuáles son las posibles diferencias entre las inhumaciones neandertales y las de los humanos modernos.

Con respecto a la distribución espacial de las sepulturas, la mayor parte del grupo neandertal se localiza en el interior de las grutas, a excepción de Tabun I y Amud I, ambos en la entrada. Los únicos restos de humanos anatómicamente modernos (Qa- fzeh y Skhul), se encuentran enterrados al aire libre. Sin embargo, no es probable que esta tendencia refleje un comportamiento diferencial entre ambas especies de homínidos, ya que Skhul es un pequeño abrigo, y en el interior de la cueva de Qafzeh los restos óseos recuperados son realmente escasos debido a alteraciones geoquímicas (Farrand, 1979; Vandermeersch, 1981). También se han realizado estudios acerca de la colocación y orientación de los cadáveres (Defleur, 1993; Smirnov, 1989), pero no se aprecia ninguna tendencia clara que diferencie a los enterramientos de los neandertales de los de humanos modernos (Tillier et alii, 1991).

Según Louwe (1989), los enterramientos por sí mismos no ofrecen mucha información sobre el pensamiento abstracto, y es en realidad la presencia de ofrendas el mejor criterio para diferenciar una conducta ritual de una simplemente higiénica. Sin embargo, en el Próximo Oriente el número de objetos a los que se pueda dar una connotación simbólica es pequeño.A parte de la mandíbula de jabalí entre las manos de SkhulV, contamos con unas astas de Dama mesopotamica junto a Qafzeh 11 y una mandíbula de cérvido acompañando a Amud 7. También se recogieron fragmentos de ocre muy numerosos en torno a Qafzeh 8, Qafzeh 9-10 y Qafzeh 11, a los que se le puede sumar el supuesto ajuar a Shanidar I, la mandíbula de cérvido junto a Shanidar V, y la pretendida ofrenda floral de Shanidar IV (Solecki, 1971; Leroi-Gourhan, 1975, 1989, 1999; en contra Gargett, 1989; Sommer, 1999), así como el descubrimiento de Dederiyeh I, en el que se documentó una pieza de sílex situada sobre el pecho del cadáver (Akazawa et alii, 1995). De todos estos ejemplos, sólo los restos asociados a Qafzeh 11 y SkhulV han sido normalmente considerados como verdaderas ofrendas, mientras que algunos autores (p.e. Lindly y Clark, 1990) ni siquiera aceptan la validez de estas últimas. De hecho, y según estos investigadores, la presencia de Sus scrofa en toda la secuencia de Skhul, unido a la perturbación de la tumba, indica que la mandíbula asociada a SkhulV no fue aportada antrópicamente. Lindly y Clark interpretan de igual forma las astas de Dama mesopotamica junto a Qafzeh 11, asegurando que se trata de restos de la ocupación del nivel XXII. Sin embargo, estas sugerencias pueden ser rebatidas; en el caso de Qafzeh, y aunque es cierto que el ciervo abunda en los suelos de ocupación del yacimiento, aparece siempre muy fragmentado, por lo que no debe ser casualidad que las únicas astas completas representadas en la se- 
cuencia sean las asociadas a Qafzeh 11 (Bar-Yosef et alii, 1990). Con respecto a Skhul, el argumento es el mismo; la mandíbula de jabalí se recuperó intacta del contexto de SkhulV, mientras que en el resto del registro Sus scrofa aparece totalmente fragmentado (Hovers et alii, 1995).

En realidad, hasta el descubrimiento de Amud 7 y su mandíbula de cérvido asociada (Hovers et alii, 1995, 2000; Rak et alii, 1994), se pensaba que únicamente Qafzeh 11 y Skhul V poseían verdaderas ofrendas. Esto es de especial relevancia, ya que de ese modo se interpretaba que el comportamiento simbólico en Próximo Oriente era patrimonio exclusivo de los humanos anatómicamente modernos (Bar-Yosef, 1988, 1989; Chase y Dibble, 1987; Tillier et alii, 1991, etc). Sin embargo, los nuevos descubrimientos de Amud 7 y los niños de Dederiyeh, avalados por otros ejemplos como la pieza grabada de Quneitra (Marshack, 1996), quizás hagan necesario un replanteamiento de las interpretaciones tradicionales, que habitualmente han rechazado cualquier tipo de manifestación simbólica en el comportamiento de los neandertales de la región.

Como venimos proponiendo en este trabajo, el patrón conductual tanto de humanos modernos como de neandertales debió ser exactamente el mismo a comienzos del último glacial. En Klasies River Mouth o Border Cave (Sudáfrica), por citar otros casos, humanos anatómicamente modernos no presentan un comportamiento simbólico distinto al documentado en nuestra región de estudio (Klein, 1995, 1998). Además, idéntica situación se reproduce entre los primeros humanos modernos del Paleolítico superior inicial del Próximo Oriente, ya que el número de sepulturas de este periodo es realmente escaso, por no decir anecdótico. De hecho, en esta zona sólo se conoce el posible enterramiento de Egbert, una joven anatómicamente moderna localizada en los niveles del Paleolítico superior inicial de Ksar Akil (Bergman y Stringer, 1989). La verdadera explosión simbólica y artística se produjo mucho después, hacia el 20-15.000 BP, y por tanto no se pueden discriminar tan sólo por el taxón diferentes comportamientos simbólicos 40.000 años antes. Es más, en el área del Próximo Oriente la citada revolución artística es todavía más tardía, en el Natufiense, por lo que no parece posible hacer una correlación automática entre el inicio del Paleolítico superior y una supuesta eclosión de las manifestaciones simbólicas (Belfer-Cohen, 1988). Cabría especular sobre si el rechazo a la existencia de sepulturas y el simbolismo implícito en ellas no se debe más a un prejuicio de los investigadores que a una realidad constatada. Como indican BelferCohen y Hovers (1992), nadie cuestiona que los natufienses enterraban a sus muertos. Y sin embargo, según estas autoras, en el Natufiense tampoco parecen existir patrones claros de enterramiento, ni de edad, ni de presencia de ofrendas, ni de posición de los cuerpos. En Hatula se recuperó el esqueleto de una joven natufiense (Ronen y Lechevallier, 1985), y nadie duda que fue objeto de un enterramiento intencionado y ritual. Pues bien, su posición es exactamente idéntica a la de Amud I; descansa sobre su costado izquierdo con los miembros flexionados y sin ningún tipo de objetos asociados. Sin embargo, y como hemos visto, muchos autores eliminan de Amud I cualquier tipo de comportamiento simbólico, conducta que sin embargo no niegan a sus vecinos anatómicamente modernos de Qafzeh y Skhul. Es evidente por tanto que existe en muchos investigadores una base teórica preconcebida que diferencia categóricamente a neandertales y modernos, negando de este modo a los primeros los atributos básicos que se conceden al Homo sapiens sapiens. Dibble y Chase (1993) hablan de la "evidencia negativa", esto es, que si no se puede demostrar que los neandertales poseían un comportamiento simbólico, éste no se puede presuponer. Estando absolutamente de acuerdo con ellos, no entendemos sin embargo por qué se dota a los humanos anatómicamente modernos del Pleistoceno superior inicial de tal conducta, cuando la propia evidencia arqueológica demuestra que, al menos en el plano simbólico, éstos se comportaron igual que sus contemporáneos neandertales.

\section{CONCLUSIONES}

A lo largo de las páginas precedentes, hemos tratado de abordar desde una perspectiva crítica las hipótesis que diferencian el comportamiento de humanos modernos y neandertales a partir de los restos arqueológicos. Nuestro estudio se ha restringido a un momento concreto, el Pleistoceno superior inicial, y a una región bien delimitada, el Próximo Oriente. En contra de la tendencia de algunos investigadores, en ningún momento pretendemos generalizar nuestras conclusiones para el resto del registro euroasiático. Es evidente que el Würm II europeo, momento en el que de nuevo volvemos a constatar una posible interrelación entre humanos

\section{T. P., 58, n. $^{\circ} 1,2001$}


modernos y neandertales, responde a una problemática absolutamente distinta a la que aquí tratamos. En sólo 10.000 años, los neandertales desaparecieron de Eurasia, y hacia el $30.000 \mathrm{BP}$, los últimos arcaicos se extinguían en el sur de la Península Ibérica. Es lícito pensar entonces, tal y como está consensuado por la mayor parte de la comunidad científica, que los humanos modernos del Auriñaciense disponían de alguna ventaja adaptativa, ya fuera física, biológica o probablemente tecnológica, que les permitió sobrevivir a los neandertales. Sin embargo, no es nuestro objetivo aquí analizar estos acontecimientos y buscar el por qué de la sustitución de poblaciones. Durante décadas, y especialmente por autores anglosajones (Binford, 1985; Stringer y Gamble 1993; Stiner 1994; Mellars, 1998, etc), se ha defendido que en términos adaptativos los neandertales fueron inferiores a los humanos modernos. No obstante, el ejemplo del Próximo Oriente no sugiere esto, al menos en lo que se refiere al Pleistoceno superior inicial; ni la tecnología de unos y otros grupos, ni los patrones de asentamiento, ni la fauna representada, y ni siquiera el comportamiento simbólico, permiten establecer diferencias entre la conducta de humanos modernos y neandertales. Este hecho comienza a ser asumido por los investigadores, que cada vez en mayor número aceptan que la aparición de Homo sapiens sapiens hace más de 100.000 años no fue paralela a una revolución cultural (Klein, 1995, 1998; Clark y Lindly 1989; Kaufman 1999, etc). Ni los primeros humanos modernos del Próximo Oriente ni los deAfrica meridional presentan diferencias conductuales o tecnológicas con respecto a otros grupos humanos del Pleistoceno superior inicial. Esto es esencial, insistimos, para eliminar de una vez por todas el viejo paradigma de que la aparición de los primeros humanos modernos trajo consigo cambios radicales en las estrategias económicas y simbólicas. La revolución tecnológica y conductual de los humanos fue muy posterior, y debe explicarse por otras causas alternativas.

Así, y en lo que se refiere al Pleistoceno superior inicial en el Próximo Oriente, parece que la mal llamada supremacía evolutiva de los humanos modernos no se aprecia en ningún caso. Para el período y la región que hemos estudiado, podríamos ahora hacernos de nuevo la pregunta que da título a este trabajo: ¿existieron en realidad diferencias conductuales entre los humanos modernos y los neandertales? Tratándose de distintos grupos humanos, lo más probable es que sí las hubiera. Ahora bien, en base a la industria, la fauna y los propios restos humanos que nos han proporcionado los yacimientos palestinos, tales diferencias no pueden demostrarse arqueológicamente. Hasta que no dispongamos de más datos, y sobre todo, de nuevas hipótesis de trabajo, debemos asumir que durante el Pleistoceno superior inicial del Próximo Oriente, los humanos anatómicamente modernos y los neandertales compartieron similares (sino iguales) modelos de comportamiento.

\section{BIBLIOGRAFÍA}

Akazawa, T.; Muhesen, S.; Dodo, Y.; Mizoguchi, Y.; Abe, Y.; Nishiaki, Y.; Ohta, S.; Oguchi, T. y Haydal, J. (1995): "Neanderthal infant burial from the Dederiyeh Cave in Syria". Paléorient, 21(2): 77-86.

Akazawa, T.; Muhesen, S.; Ishida, H.; Kondo, O.; GrigGo, C. (1999): "New discovery of a Neanderthal child burial from the Dederiyeh Cave in Syria». Paléorient, 25(2): 129-142.

Alperson, N; Barzilai, O.; DAG, D.; Hartman, G. y MatsKEVICH, Z. (2000): "The age and context of the Tabun I skeleton: a reply to Schwarcz et al". Journal of $\mathrm{Hu}$ man Evolution, 38: 849-853.

Arensburg, B. y Belfer-Cohen, A. (1998): “Sapiens and Neandertals: Rethinking the Levantine Middle Paleolithic Hominids". En T. Akazawa, K. Aoki y O. BarYosef (eds.): Neandertals and Modern Humans in Western Asia. Plenum Press. New York: 311-322.

BAR-Yosef, O. (1988): "Evidence for Middle Palaeolithic Symbolic Behavior: A Cautionary Note". En M. Otte (ed.): L'Homme de Néandertal. Actes du colloque international de Liège (4-7 décembre 1986). En O. BarYosef (coord.): La Pensée, 5. Service de Prehistoire. Université de Liège: 11-16.

- (1989): "Upper Pleistocene cultural stratigraphy in southwest Asia". En E. Trinkaus (ed.): The Emergence of Modern Humans. Biocultural Adaptations in the later Pleistocene. Cambridge University Press. Cambridge: $154-180$.

- (1992): "Middle Paleolithic Adaptations in the Mediterranean Levant". EnT. Akazawa, K.Aoki yT. Kimura (eds.): The Evolution and Dispersal of Modern $\mathrm{Hu}$ mans in Asia. Hokusen-sha. Tokio: 189-216.

- (1994): "The Contributions of Southwest Asia to the Study of the Origin of Modern Humans". En M.H. Nitecki y D.V. Nitecki (eds.): Origins of Anatomically Modern Humans. Plenum Press. New York: 24-66.

- (1998): "The Chronology of the Middle Paleolithic of the Levant". En T. Akazawa, K. Aoki y O. Bar-Yosef (eds.): Neandertals and Modern Humans in Western Asia. Plenum Press. New York: 39-56.

Bar-Yosef, O. y CALlander, J. (1999): “The woman from

T. P., 58, n. ${ }^{\circ} 1,2001$ 
Tabun: Garrod's doubts in historical perspective". Journal of Human Evolution, 37: 879-885.

BAR-Yosef, O.; Lieberman, D. y Shea, J.J. (1990): Comentarios a "Symbolism and Modern Human Origins". Current Anthropology, 31(3): 240-241.

Bar-Yosef, O. y VANDERmeErsch, B. (1981): "Notes concerning the possible age of the Mousterian layers at Qafzeh Cave". En J. Cauvin y P. Sanlaville (eds.): Préhistoire du Levant: Chronologie et organisation de l'espace depuis les origines jusqu'au VI e millénaire. CNRS. París: 281-285.

Bar-Yosef, O.; Vandermeersch, B.; Arensburg, B.; Belfer-Cohen, A.; Goldberg, P.; Laville, H.; Meignen, L.; RaK, Y.; SPeth, J.D.; Tchernov, E.; TIllier, A.-M. y Weiner, S. (1992): "The Excavations in Kebara Cave, Mt. Carmel". Current Anthropology, 33(5): 497-550.

Belfer-CoHen, A. (1988): "The Appearance of Symbolic Expression in the Upper Pleistocene of the Levant as compared to Western Europe". En M. Otte (ed.): L'Homme de Néandertal.Actes du colloque international de Liège (4-7 décembre 1986). En O. Bar-Yosef (coord.): La Pensée, 5. Service de Prehistoire. Université de Liège: $25-29$.

- (1993): Comentarios a "The Rise and Fall of Seasonal Mobility among Hunter-Gatherers. The Case of the Southern Levant". Current Anthropology, 34(5): 614615.

Belfer-Cohen, A. y Hovers, E. (1992): "In the Eye of the Beholder: Mousterian and Natufian Burials in the Levant". Current Anthropology, 33(4): 463-71.

Bergman, C.A. y STRINGER, C.B. (1989): "Fifty years after: Egbert, an Early Upper Palaeolithic Juvenile from Ksar Akil, Lebanon". Paléorient, 15(2): 99-112.

BINFORD, L.R. (1980): "Willow smoke and dogs' tails: Hunter-gatherer settlement systems and archaeological site formation". American Antiquity, 45: 4-20.

- (1981): Bones. Ancient men and modern myth. Academic Press. New York.

- (1983): "Long-term Land-Use Patterning: Some Implications for Archaeology". En L.R. Binford (ed.): Working at Archaeology. Academic Press. New York: 379386.

- (1985): "Human ancestors: changing views of their behaviour". Journal of Anthropological Archaeology, 4: 292-327.

BINFORD, S.R. (1968): "Early Upper PleistoceneAdaptations in the Levant". American Anthropologist, 70(4): 707-717.

BocQuet-ApPel, J-P. y Demars, P.Y. (2000): "Neanderthal contraction and modern human colonization of Europe”. Antiquity, 74: 544-552.

BoutiÉ, P. (1989): "Etude technologique de l'industrie moustérienne de la Grotte de Qafzeh (près de Nazareth, Isräel)". En O. Bar-Yosef y B. Vandermeersch (eds.): Investigations in South Levantine Prehistory. B.A.R. International Series. Oxford, 497: 213-230.
BRown, T. y MolnaR, S. (1990): "Interproximal grooving and task activity in Australia". American Journal of PhysicalAnthropology, 81: 545-553.

Chase, P.G. y Dibble, H.L. (1987): "Middle Paleolithic Symbolism: A Review of Current Evidence and Interpretations". Journal of AnthropologicalArchaeology, 6(3): 263-296.

Clark, G.A. y Lindly, J. (1989): “The Case for Continuity: Observations on the Biocultural Transition in Europe andWestern Asia". En P. Mellars y C. Stringer (eds.): The Human Revolution. Behavioral and Biological Perspectives on the Origins of Modern Humans. Edinburgh University Press. Edinburgh: 626-676.

Copeland, L. (1975): "The Middle and Upper Palaeolithic of Lebanon and Syria in the light of recent research". En F. Wendorf y A. Marks (eds.): Problems in Prehistory, North Africa and the Levant. Southern Methodist University Press. Dallas: 317-350.

- (1981): "Chronology and Distribution of the Middle Paleolithic, as known in 1980, in Lebanon and Syria". En J. Cauvin y P. Sanlaville (eds.): Préhistoire du Levant: Chronologie et organisation de l'espace depuis les origines jusqu'au VI e millénaire. CNRS. París: 239-263.

- (1983): "The Palaeolithic Industries at Adlun". En D.A. Roe (ed.): Adlun in the Stone Age. The Excavations of D.A.E. Garrod in the Lebanon, 1958-1963. B.A.R. International Series. Oxford, 159, I-II: 89-365.

DAVIS, S. (1977): “The ungulate remains from Kebara Cave". Eretz Israel, 13: 150-63.

DaVIS, S.; RABINOVICH, R. y GoREN-INBAR, N. (1988): "Quaternary extinctions and population increase in Western Asia: The Animal Remains from Biq'at Quneitra”. Paléorient, 14(1): 95-105.

Defleur, A. (1993): Les Sépultures Moustériennes. CNRS. París.

D’Errico, F.; Zilhão, J.; Julien, M.; Baffier, D. y PeleGRÍN, J. (1998): "Neanderthal Acculturation in Western Europe?A Critical Review of the Evidence and Its Interpretation". CurrentAnthropology, 39, suplemento de junio: 1-44.

Dibble, H.L. y Chase, P.G. (1993): "On Mousterian and Natufian Burials in the Levant". Current Anthropology, 34(2): 170-172.

Evins, M.A. (1982): "The Fauna from Shanidar Cave: Mousterian Wild Goat Exploitation in Northeastern Iraq". Paléorient, 8(1): 37-58.

FARRAND, W.R. (1979): "Chronology and paleoenvironment of the Levantine prehistoric sites as seen from sediment studies". Journal of Archaeological Science, 6: 369-392.

GARGETT, R.H. (1989): "Grave Shortcomings. The Evidence for Neandertal Burial". Current Anthropology, 30(2): 157-190.

- (1999): "Middle Palaeolithic burial is not a dead issue: the view from Qafzeh, Saint-Césaire, Kebara, Amud,

T. P., 58, n. $^{\circ} 1,2001$ 
and Dederiyeh". Journal of Human Evolution, 37: 27 90.

- (2000): "A response to Hovers, Kimbel and Rak's argument for the purposeful burial of Amud 7". Journal of Human Evolution, 39: 261-266.

GARRARD, A.N. (1982): “The Environmental Implications of a Re-Analysis of the Large Mammal Fauna from the Wadi El-Mughara Caves, Palestine". En J.L. Bintliff y W. Van Zeist (eds.): Palaeoclimates, Palaeoenvironments and Human Communities in the Eastern Mediterranean in later Prehistory. B.A.R. International Series. Oxford, 133: 165-187.

- (1983): "The Palaeolithic Faunal Remains from Adlun and their Ecological Context", En D.A. Roe (ed.): Adlun in the Stone Age. The Excavations of D.A.E. Garrod in the Lebanon, 1958-1963. B.A.R. International Series. Oxford, 159, II: 397-409.

GARrod, D.A.E. y BATE, D.M.A. (1937): The Stone Age of Mount Carmel. Excavation at the Wady El-Mughara, 1. Clarendon Press. Oxford.

Gilead, I. y Grigson, G. (1984): "Far'ah II: A Middle Palaeolithic Open-Air Site in the Northern Negev, Israel". Proceedings of the Prehistoric Society, 50: 71-97.

Goldberg, P. y Bar-Yosef, O. (1998): "Site Formation Processes in Kebara and Hayonim Caves and Their Significance in Levantine Prehistoric Caves". En T. Akazawa, K.Aoki y O. Bar-Yosef (eds.): Neandertals and Modern Humans in Western Asia. Plenum Press. New York: 107-125.

Goren-Inbar, N. y Belfer-Cohen, A. (1998): “The TechnologicalAbilities of the Levantine Mousterians: $\mathrm{Cul}$ tural and Mental Capacities". EnT. Akazawa, K.Aoki y O. Bar-Yosef (eds.): Neandertals and Modern $\mathrm{Hu}$ mans in Western Asia. Plenum Press. New York: 205221.

GrÜn, R.; STringer, C.B. y Schwarcz, H.P. (1991): "ESR dating of teeth from Garrod's Tabun Cave collection". Journal of Human Evolution, 20: 231-248.

GRÜN, R.; STRINGER, C. (2000): “Tabun revisited: revised ESR chrnology ad new ESR and U-series analyses of dental material from Tabun C1". Journal of Human Evolution, 39: 601-612.

HenRy, D.O. (1992): "Transhumance During the Late Levantine Mousterian". En H.L. Dibble y P. Mellars (eds.): The Middle Paleolithic: Adaptation, behavior, and variability. University of Pennsylvania Museum. Philadelphia: 143-62.

- (1995): Prehistoric Cultural Ecology and Evolution. Insights from Southern Jordan. Plenum Press. New York.

- (1998): "The Middle Paleolithic of Jordan”. En D.O. Henry (ed.): The Prehistoric Archaeology of Jordan. B.A.R. International Series. Oxford, 705: 23-38.

- (1998b): Comentarios a "Neandertal and Early Modern Human Behaviora Variability. A Regional-Scale Approach to Lithic Evidence for Hunting in the Levan- tine Mousterian”. Current Anthropology, 39, suplemento de junio: 64-65.

HollidAY, T.W. (2000): "Evolution at the Crossroads: Modern Human Emergence in Western Asia". American Anthropologist, 102(1): 54-68.

Hovers, E. (1998): "The Lithic Assemblages of Amud Cave: Implications for Understanding the End of the Mousterian in the Levant". En T. Akazawa, K. Aoki y O. Bar-Yosef (eds.): Neandertals and Modern Humans in Western Asia. Plenum Press. New York: 143-163.

Hovers, E.; Kimbel, W.H. y RaK, Y. (2000): "The Amud 7 skeleton - still a burial. Response to Gargett". Journal of Human Evolution. 39: 253-260.

Hovers, E.; RAK, Y.; LAVI, R. y KimBel, W.H. (1995): “Hominid Remains from Amud Cave in the Context of the Levantine Middle Paleolithic". Paléorient, 21(2): 47 61.

JelineK, A.J. (1981): "The Middle Paleolithic in the Southern Levant from the perspective of the Tabun cave". En J. Cauvin y P. Sanlaville (eds.): Préhistoire du Levant: Chronologie et organisation de l'espace depuis les origines jusqu'au VI e millénaire. CNRS. París: 265-280.

- (1982): “The Middle Palaeolithic in the southern Levant with comments on the appearance of modern Homo sapiens". EnA. Ronen (ed.): The Transition from the Lower to the Middle Palolithic and the Origin of Modern Man. B.A.R. International Series. Oxford, 151: $57-104$

KaUfman, D. (1999): Archaeological Perspectives on the Origins of Modern Humans. A View from the Levant. Bergin and Garvey. Connecticut.

KleiN, R.G. (1995): "Anatomy, Behavior, and Modern Human Origins". Journal of World Prehistory, 9(2): 167-198.

- (1998): "Why Anatomically Modern People Did Not Disperse from Africa 100,000 Years Ago". En T. Akazawa, K. Aoki y O. Bar-Yosef (eds.): Neandertals and Modern Humans in Western Asia, Plenum Press. New York: 509-521.

Kozlowski, J.K. у Otте, M. (2000): "La formation de l'Aurignacien en Europe". L'Anthropologie, 104: 3-15.

KuHN, S.L. (1998): Comentarios a "Neandertal and Early Modern Human Behavioral Variability. A RegionalScale Approach to Lithic Evidence for Hunting in the Levantine Mousterian". Current Anthropology, 39, suplemento de junio: 66-67.

Leroi-Gourhan, A. (1975): "The flowers found with Shanidar IV, a Neanderthal burial in Iraq". Science, 190: 562-64.

- (1989): Comentarios a "Grave Shortcomings. The Evidence for Neandertal Burial”. Current Anthropology, 30(2): 182.

- (1999): "Shanidar et ses fleurs". Paléorient, 24(2): 7988.

Lieberman, D.E. (1993): "The Rise and Fall of Seasonal

T. P., 58, n. $^{\circ} 1,2001$ 
Mobility among Hunter-Gatherers. The Case of the Southern Levant". Current Anthropology, 34(5): 599631.

- (1998): "Neandertal and Early Modern Human Mobility Patterns: Comparing Archaeological and Anatomical Evidence". En T. Akazawa, K. Aoki y O. Bar-Yosef (eds.): Neandertals and Modern Humans in Western Asia. Plenum Press. New York: 263-275.

LIEBERMAN, D.E. y SHEA, J.J. (1994): "Behavioral Differences between Archaic and Modern Humans in the Levantine Mousterian”. American Anthropologist, 96(2): 300-332.

LINDLY, J.M. y ClARK, G.A. (1990): "Symbolism and Modern Human Origins". Current Anthropology, 31(3): 233-261.

Louwe, L.P. (1989): “On the Evidence for Neandertal Burial”. Current Anthropology, 30(3): 322-330.

ManN, A. (1995): "Modern Human Origins: Evidence from the Near East". Paléorient, 21(2): 35-46.

MAREAN, C.W. (1998): "A critique of the evidence for scavenging by Neandertals and early modern humans: new data from Kobeh Cave (Zagros Mountains, Iran) and Die Kelders Cave I Layer 10 (South Africa)". Journal of Human Evolution, 35: 115-136.

Marean, C.W. y Assefa, Z. (1999): "Zooarcheological Evidence for the Faunal Exploitation Behavior of Neandertals and Early Modern Humans". Evolutionary Anthropology, 8(1): 22-37.

Marean, C.W. y KIM, S.Y. (1998): "Mousterian LargeMammal Remains from Kobeh Cave". Current Anthropology, 39, suplemento de junio: 79-113.

MarKs, A.E. (1998): Comentarios a "Pointes ou outils triangulaires? Données fonctionnelles dans le Moustérien Levantin". Paléorient, 24(1): 18-20.

Marshack, A. (1996): “A Middle Paleolithic Symbolic Composition from the Golan Heigths: The Earliest Known Depictive Image". Current Anthropology, 37(2): 357-65.

Meignen, L. (1998): "Hayonim Cave Lithic Assemblages in the Context of the Near Eastern Middle Paleolithic: A Preliminary Report”. En T. Akazawa, K. Aoki y O. Bar-Yosef (eds.): Neandertals and Modern Humans in Western Asia. Plenum Press. New York: 165-180.

Mellars, P. (1998): "The Impact of Climatic Changes on the Demography of Late Neandertal and Early Anatomically Modern Populations in Europe". En T. Akazawa, K.Aoki y O. Bar-Yosef (eds.): Neandertals and Modern Humans in Western Asia. Plenum Press. New York: 493-507.

Mellars, P; Otte, M.; Straus, L.G; Zilhão, J. y D’Errico, F. (1999): “The Neanderthal Problem Continued". Current Anthropology, 40 (3): 341-364.

Mercier, N.; Valladas, H.;Bar-Yosef, O.; Stringer, C. y JORON, J.L. (1993): "Thermoluminescence date for the Mousterian Burial Site of Es-Skhul, Mount Carmel". Journal of Archaeological Science, 20(2): 169-74.
Mercier, N.; Valladas, H.; Valladas, G.; Reyss, J.L.; JelineK, A.; Meignen, L. y Joron, J.L. (1995): “TL Dates of Burnt Flints from Jelinek's Excavations at Tabun and their Implications". Journal of Archaeological Science, 22(4): 495-509.

Millard, A.R. y Pike, A.W.G. (1999): "Uranium-series dating of the Tabun Neanderthal: a cautionary note". Journal of Human Evolution, 36: 581-585.

Molnar, S.; Richards, L.; McKeE, J. y Molnar, I. (1989): "Tooth wear in Australian aboriginal populations from the river Murray valley". American Journal of Physical Anthropology, 79: 185-196.

Monigal, K.; Marks, A.E.; Demidenko, Y.E.; Usik, V.I.; RINK, W.J.; Schwarcz, H.P.; FERring, C.R. y McKinNEY, C.R. (1997): "Nouvelles decouvertes de restes humains au site Paleolithique moyen de Starosele, Crimee (Ukraine)". Préhistoire Européene, 11: 11-31.

NIEWOEHNER, W.A. (2001): "Behavioral references from the Skhul/Aofreh early modern human hand remains". Proceedings of the National Academy of Sciences, 98(6): 2979-2984.

NisHIAKI, Y. (1998): “The Paleolithic and Neolithic of Syria: An Overview with Reference to Jordanian Prehistory". En D.O. Henry, (ed.): The Prehistoric Archaeology of Jordan. B.A.R. International Series. Oxford, 705: 195207.

OlsZEwski, D.I. (1993): Comentarios a "The Rise and Fall of Seasonal Mobility among Hunter-Gatherers. The Case of the Southern Levant". Current Anthropology, 34(5): 617-618.

Perkins, D. (1964): "Prehistoric Fauna From Shanidar, Iraq". Science, 144: 1565-1566.

Plisson, H. y Beyries, S. (1998): "Pointes ou outils triangulaires? Données fonctionnelles dans le Moustérien Levantin". Paléorient, 24(1): 5-24.

QuAm, R.M. y Sмiтh, F.H. (1998): "A Reassessment of the Tabun C2 Mandible”. En T. Akazawa, K. Aoki y O. BarYosef (eds.): Neandertals and Modern Humans in Western Asia. Plenum Press. New York: 405-421.

RABINOVICH, R. (1990): "Taphonomic Research on the Faunal Assemblage from the Quneitra Site". En N. GorenInbar (ed.): Quneitra: A Mousterian Site on the Golan Heights. Qedem, Monographs of the Institute of Archaeology, The Hebrew University of Jerusalem. Jerusalem, 31: 189-219.

- (1998): "Drowning in Numbers. Gazelles Dominance and Body Size Groups in the Archaeozoological Record". En H. Buitenhuis, L. Bartosiewicz y A.M. Choyke (eds.): Archaeozoology of the Near East III. Proceedings of the Third International Symposium on the Archaeozoology of Southwestern Asia and Adjacent Areas. ARC Publications, Groningen: 45-71.

RABinOvich, R. yTcheRnov, E. (1995): “Chronological, Paleoecological andTaphonomicalAspects of the Middle Paleolithic Site of Qafzeh, Israel". En H. Buitenhuis y H.-P. Uerpmann (eds.): Archaeozoology of the Near

T. P., 58, n. ${ }^{\circ} 1,2001$ 
East II. Proceedings of the Second International Symposium on theArchaeozoologyof SouthwesternAsiaand AdjacentAreas. Backhuys Publishers. Leiden: 5-44.

RAK, Y. (1998): "Does Any Mousterian Cave Present Evidence of Two Hominid Species?”. En T. Akazawa, K. Aoki y O. Bar-Yosef (eds.): Neandertals and Modern Humans in Western Asia. Plenum Press. New York: 353-366.

RaK, Y.; Kimbel, W.H. y Hovers, E. (1994): “A Neandertal infant from Amud Cave, Israel". Journal of Human Evolution, 26: 313-324.

Ronen, A. y LeCheVAlLIER, M. (1985): “The Natufian-Early Neolithic site of Hatula, near Latrun, Israel". Quartär, 35-36: 141-164.

Schwarcz, H.P. y RinK, W.J. (1998): "Progress in ESR and U-Series Chronology of the Levantine Paleolithic". En T. Akazawa, K. Aoki y O. Bar-Yosef (eds.): Neandertals and Modern Humans in Western Asia. Plenum Press. New York: 57-67.

Schwarcz, H.P.; Simpson, J.J. y Stringer, C.B. (1998): "Neanderthal skeleton from Tabun: U-series data by gamma-ray spectrometry". Journal of Human Evolution, 35: 635-645.

ShEA, J.J. (1988): "Spear Points fromt the Middle Paleolithic of the Levant". Journal of Field Archaeology, 15: 441-450.

- (1989): “A Functional Study of the Lithic Industries Associated with Hominid Fossils in the Kebara and Qafzeh Caves, Israel". En P. Mellars y C. Stringer (eds.): The Human Revolution: Behavioral and Biological Perspectives on the Origins of Modern Humans. Edinburgh University Press. Edinburgh: 611-625.

- (1995): "Behavioral Factors Affecting the Production of Levallois Points in the Levantine Mousterian". En H.L. Dibble y O. Bar-Yosef (eds.): The Definition and Interpretation of Levallois Technology. Prehistory Press. Madison: 279-292.

- (1998): "Neandertal and Early Modern Human Behavioral Variability. A Regional-ScaleApproach to Lithic Evidence for Hunting in the Levantine Mousterian". Current Anthropology, 39, suplemento de junio: 45-78.

Smirnov, Y. (1989): "Intentional Human Burial: Middle Paleolithic (Last Glaciation) Beginnings". Journal of World Prehistory, 3(2): 199-233.

Solecki, R.S. (1971): Shanidar. The First Flower People. Alfred Knopf. New York.

Sommer, J.D. (1999): "The Shanidar IV "Flower Burial": a Reevaluation of Neanderthal Burial Ritual". Cambridge Archaeological Journal, 9(1): 127-137.

Speth, J.D. y Tchernov, E. (1998): "The Role of Hunting and Scavenging in Neandertal Procurement Strategies: New Evidence from Kebara Cave (Israel)". EnT. Akazawa, K. Aoki y O. Bar-Yosef (eds.): Neandertals and Modern Humans in Western Asia. Plenum Press. New York: 223-239.
Stiner, M.C. (1994): Honor Among Thieves: A Zooarchaeological Study of Neandertal Ecology. Princeton University Press. Princeton.

Stiner, M.C.; Munro, N.D.; Surovell, T.A.; TChernov, E. y Bar-Yosef, O. (1999): "Paleolithic Population Growth Pulses Evidenced by Small Animal Exploitation". Science, 283:190-194.

Stiner, M.C.; Munro, N.D. y Surovell, T.A. (2000): “The Tortoise and the Hare: Small-Game Use, the BroadSpectrum Revolution, and Paleolithic Demography". Current Anthropology, 41(1): 39-73.

STINER, M.C. y TCHERnov, E. (1998): "Pleistocene Species Trends at Hayonim Cave: Changes in Climate versus Human Behavior". En T. Akazawa, K. Aoki y O. BarYosef (eds.): Neandertals and Modern Humans in Western Asia. Plenum Press. New York: 241-262.

Stringer, C.B. (1998): "Chronology and Biogeographic Perspectives on Later Human Evolution". En T. Akazawa, K. Aoki y O. Bar-Yosef (eds.): Neandertals and Modern Humans in Western Asia. Plenum Press. New York: 29-37.

StRINGER, C.B. y GAMBle, C. (1993): In search of the Neanderthals: solving the puzzle of human origins. Thames and Hudson. London.

TAKAI, F. (1970): "Fossil Mammals from the Amud Cave". En H. Suzuki y F. Takai (eds.): The Amud Man and his Cave Site. Academic Press of Japan. Tokio: 53-76.

TChernov, E. (1992): "Biochronology, Paleoecology, and Dispersal Events of Hominids in the Southern Levant". En T. Akazawa, K. Aoki y T. Kimura (eds.): The Evolution and Dispersal of Modern Humans inAsia. Hokusen-sha. Tokyo: 149-188.

- (1998): "The Faunal Sequence of the Sotuhwest Asian Middle Paleolithic in Relation to Hominid Dispersal Events". EnT. Akazawa, K.Aoki y O. Bar-Yosef (eds.): Neandertals and Modern Humans in Western Asia. Plenum Press. New York: 77-90.

Tillier, A-M.; Arensburg, B.; RaK, Y. y Vandermeersch, B. (1988): "Les sépultures néandertaliennes du Proche Orient: état de la question”. Paléorient, 14(2): 130-136.

Tillier, A-M.; Arensburg, B.; VAndermeersch, B. y RaK, Y. (1991): "L'apport de Kébara à la palethnologie funéraire des néanderthaliens du Proche-Orient". En O. Bar-Yosef y B. Vandermeersch (eds.): Le squelette moustérien de Kébara 2. CNRS. París: 89-95.

Torre, I. de la y Domínguez-Rodrigo, M. (2000): "El Paleolítico medio en el Próximo Oriente: una síntesis regional". Complutum, 11: 9-27.

TrinkAus, E. (1983): The Shanidar Neandertals. Academic Press, New York.

- (1989): “The Upper Pleistocene Transition". En E. Trinkaus (ed.): The Emergence of Modern Humans. Biocultural adaptations in the Later Pleistocene. Cambridge University Press. Cambridge: 42-66.

- (1992): "Morphological contrasts between the NearEastern Qafzeh-Skhül and late archaic human samples: 
Grounds for a behavioral difference?". En T. Akazawa, K. Aoki y T. Kimura (eds.): The evolution and dispersal of modern humans in Asia. Hokusen-sha. Tokyo: 277-294.

- (1993): Comentarios a "The Rise and Fall of Seasonal Mobility among Hunter-Gatherers. The Case of the Southern Levant". Current Anthropology, 34(5): 620622.

- (1995): "Near Eastern Late Archaic Humans". Paléorient, 21(2): 9-23.

Trinkaus, E. y RufF, C.B. (1999): "Dyaphyseal Cross-sectional Geometry of Near Eastern Middle Palaeolithic Humans: The Femur". Journal of Archaeological Science, 26: 409-424.

- (1999b): "Dyaphyseal Cross-sectional Geometry of Near Eastern Middle Palaeolithic Humans: The Tibia". Journal of Archaeological Science. 26: 1289-1300.

Trinkaus, E.; Ruff, C.B. y Churchill, S.E. (1998): “Upper Limb versus Lower Limb Loading Patterns among Near Eastern Middle Paleolithic Hominids". En T.Akazawa, K. Aoki y O. Bar-Yosef (eds.): Neandertals and Modern Humans in Western Asia. Plenum Press. New York: 391-404.

Valladas, H.; Joron, J.L.; Valladas, G.; Arensburg, B.; Bar-Yosef, O.; Belfer-Cohen, A.; Goldberg, P.; LAville, H.; Meignen, L.; RaK, Y.; Tchernov, E.; Tillier, A.-M. y VANDERMEERSCH, B. (1987): "Thermoluminescence dates for Neanderthal burial site at Kebara in Israel". Nature, 330: 159-60.
VAlladas, H.; Reyss, J.L.; JoRON, J.; Valladas, G.; BaRYosef, O. y VandermeERSCH, B. (1988): "Thermoluminescence dating of Mousterian "Proto-Cro-Magnon" remains from Israel and the origin of man". Nature, 331: 614-16.

VAlladAs, H.; MERCIER, N.; Froget, L.; Hovers, E.; JoRON, J.L.; KIMBEL, W.H. y RAK, Y. (1999): "TL Dates for the Neanderthal Site of the Amud Cave, Israel". Journal of Archaeological Science, 26: 259-268.

VANDERMEERSCH, B. (1981): Les hommes fossiles de Qafzeh (Israël). CNRS. París.

Wolf, C.A. (1988): "Analysis of Faunal Remains from Early Upper Paleolithic Sites in the Levant". En J.F. Hoffecker y C.A. Wolf (eds.): The Early Upper Paleolithic. Evidence from Europe and the Near East. B.A.R. International Series. Oxford, 437: 73-96.

Wolpoff, M.H. (1989): "The place of Neandertals in human evolution". En E. Trinkaus (ed.): The Emergence of Modern Humans. Biocultural Adaptations in the later Pleistocene. Cambridge University Press. Cambridge: 97-141.

ZilhÃo, J. y D'ERrico, F. (1999): “ The Chronology and Taphonomy of the Earliest Aurignacian and Its Implications for the Understanding of Neandertal Extinction". Journal of World Prehistory, 13(1): 1-68.

- (2000): "La nouvelle bataille aurignacienne. Une révision critique de la chronologie du Châtelperronien et de l'Aurignacien ancien". L'Anthropologie, 104: $17-50$. 